\title{
Transposable elements in phytopathogenic Verticillium spp.: insights into genome evolution and inter- and intra-specific diversification
}

Stefan G Amyotte ${ }^{1 \dagger}$, Xiaoping Tan ${ }^{2 \dagger}$, Kayla Pennerman², Maria del Mar Jimenez-Gasco ${ }^{3}$, Steven J Klosterman ${ }^{4}$, Li-Jun $\mathrm{Ma}^{5}$, Katherine F Dobinson ${ }^{1,6^{*}}$ and Paola Veronese $2^{2^{*}}$

\begin{abstract}
Background: Verticillium dahliae (Vd) and Verticillium albo-atrum (Va) are cosmopolitan soil fungi causing very disruptive vascular diseases on a wide range of crop plants. To date, no sexual stage has been identified in either microorganism suggesting that somatic mutation is a major force in generating genetic diversity. Whole genome comparative analysis of the recently sequenced strains VdLs.17 and VaMs.102 revealed that non-random insertions of transposable elements (TEs) have contributed to the generation of four lineage-specific (LS) regions in VdLs.17.

Results: We present here a detailed analysis of Class I retrotransposons and Class II "cut-and-paste" DNA elements detected in the sequenced Verticillium genomes. We report also of their distribution in other $\mathrm{Vd}$ and $\mathrm{Va}$ isolates from various geographic origins. In VdLs.17, we identified and characterized 56 complete retrotransposons of the Gypsy-, Copia- and LINE-like types, as well as 34 full-length elements of the "cut-and-paste" superfamilies Tc1/mariner, Activator and Mutator. While Copia and Tc1/mariner were present in multiple identical copies, Activator and Mutator sequences were highly divergent. Most elements comprised complete ORFs, had matching ESTs and showed active transcription in response to stress treatment. Noticeably, we found evidences of repeat-induced point mutation (RIP) only in some of the Gypsy retroelements. While Copia-, Gypsy- and Tc1/mariner-like transposons were prominent, a large variation in presence of the other types of mobile elements was detected in the other Verticillium spp. strains surveyed. In particular, neither complete nor defective "cut-and-paste" TEs were found in VaMs.102.
\end{abstract}

Conclusions: Copia-, Gypsy- and Tc1/mariner-like transposons are the most wide-spread TEs in the phytopathogens V. dahliae and V. albo-atrum. In VdLs.17, we identified several retroelements and "cut-and-paste" transposons still potentially active. Some of these elements have undergone diversification and subsequent selective amplification after introgression into the fungal genome. Others, such as the ripped Copias, have been potentially acquired by horizontal transfer. The observed biased TE insertion in gene-rich regions within an individual genome (VdLs.17) and the "patchy" distribution among different strains point to the mobile elements as major generators of Verticillium intra- and inter-specific genomic variation.

Keywords: Transposable elements, Verticillium spp., Retrotransposons, DNA transposons, Repeat-induced point mutation (RIP), TE domestication, Genome evolution

\footnotetext{
* Correspondence: katherine.dobinson@agr.gc.ca; paola_veronese@ncsu.edu

${ }^{\dagger}$ Equal contributors

${ }^{1}$ University of Western Ontario, London, ON, Canada

${ }^{2}$ Department of Plant Pathology, North Carolina State University, Raleigh, NC,

USA

Full list of author information is available at the end of the article
} 


\section{Background}

Present in the environment as free-living organisms or in symbiosis with other organisms, fungi have a major impact on human society. Besides being major contributors to the decomposition and recycling of nutrients, they are largely employed in food production, biotechnologies and agricultural systems. However, these microorganisms also cause devastating diseases, particularly of plants, representing a major threat to global food security. It is noteworthy that a significant number of plant pathogenic fungi are able to cause serious human and animal diseases, for which there are very few effective therapeutic agents.

Verticillium dahliae (Vd) and $V$. albo-atrum (Va) (Eukaryota, Fungi, Ascomycota) cause very disruptive vascular diseases in over 400 plant species, including vegetable, ornamental and tree crops [1]. These microorganisms penetrate the root system of their hosts and induce typical leaf wilt symptoms by spreading throughout the xylem vessels and disrupting water transport. Compared to $\mathrm{Va}, \mathrm{Vd}$ is generally characterized by a broader host range and geographical distribution, and the capability to produce longer lasting soil resting structures called microsclerotia. No sexual reproduction has been reported in either $\mathrm{Vd}$ or $\mathrm{Va}$, thus implicating somatic mutation as a driving force in their evolution. Comparative analysis of the recently sequenced genomes of the isolates VdLs.17 (33.8 Mb) and VaMs.102 (32.8 Mb), showed that they are $97 \%$ identical and that VdLs.17 contains regions in chromosomes three and four that are not present in the Va isolate [2]. These lineage-specific (LS) regions, which encompass about $3.5 \%$ of the fungal genome, comprise about 350 protein-coding genes and are also enriched in repetitive sequences corresponding to both previously known [3,4] and as-yet uncharacterized transposon-like sequences [2].

Transposable elements (TEs) are found in virtually every prokaryotic and eukaryotic genome where they can exert a multifaceted mutagenic activity that leads to changes in chromosome architecture, generation of new regulatory networks and increases in the protein repertoire [5-7]. On the basis of structural-functional characteristics, TEs have been separated into two major classes each comprising subclasses or orders, superfamilies, families and subfamilies [8,9].

The elements of Class I, retrotransposons, replicate by a "copy-and-paste" mechanism that generates RNA intermediates, which are subsequently reverse transcribed into double-stranded (ds) DNA by TE-encoded enzymes. These retroelements have been recently classified into five orders comprised of long terminal repeat (LTR) elements, Dictyostelium intermediate repeat sequence (DIRSs), Penelope-like elements (PLEs), and long and short interspersed nuclear elements (LINEs and
SINEs) $[8,9]$. Among the most widespread retrotransposons are the LTR superfamilies Gypsy and Copia, which include two ORFs coding for the structural virus-like protein GAG and the reverse transcriptase (RT)/integrase (INT) enzyme POL.

Class II elements, DNA transposons, are mobilized via the formation of DNA intermediates. If the intermediate is dsDNA, the transposition is called "conservative" and the elements are of the "cut-and-paste" type. Complete, alias autonomous, TEs of this type are generally delimited by terminal inverted repeats (TIRs) and encode a single transposase. The transposase binds conserved regions within the TIRs, mostly direct short tandem duplications, and catalyzes the excision of the complete element, which then reintegrates in a new genomic location. When TE mobilization proceeds via single-stranded (ss) DNA and does not involve the element excision it is defined as "replicative", a mode of transposition that characterizes the more complex Helitron- and Mavericklike transposons [10,11].

Although fungal genomes generally contain fewer repetitive sequences than do higher eukaryotes [8,12], TEs have played critical roles in the evolution of their fungal hosts and, in particular, of the phytopathogens. In the rice blast fungus, Magnaporthe oryzae, for example, TEs were found to cluster in regions having high recombination rates and gene duplication events [13]. Similarly, in the black mold Aspergillus niger, specific retrotransposonmediated recombinations have led to inversions of genomic regions [14]. In addition, host-specific toxin genes of Cochliobolus carbonum [15] and Alternaria alternata [16], as well as host-specificity genes of $M$. oryzae [17] are situated in transposon-rich regions of the genome. Because gene products conferring host-specificity can be recognized by plant host defense receptors, it has been hypothesized that this proximity could allow expansion of host range through TE-mediated gene loss [17]. In addition, TE distribution can be extremely variable between isolates of a single fungal species, and TEs have been used as markers to distinguish genetically divergent populations, and for the identification of subpopulations [18].

Introgression of mobile elements into the genome is potentially deleterious, and cells have evolved different processes for their elimination, including silencing by RNA interference and repeat-induced point (RIP) mutation [19-21]. The introduction of RIP mutations in the DNA of fungi is linked to sexual reproduction, occurring at the time of meiosis, and the process likely arose as a genome defense mechanism against the intrusion of repetitive DNA sequences. The RIP machinery recognizes duplicated sequences longer than $400 \mathrm{bp}$ and sharing more than $80 \%$ identity, and introduces in both copies C:G to T:A transitions [22]. Noticeably, RIP has been 
detected in the asexual fungi A. niger, Penicillium crysogenum and $V$. dahliae [2,23], suggesting the existence of an ancestral or cryptic sexual state in these species. Interestingly, RIP mutations in VdLs.17 affected members of the Gypsy but not of the Copia superfamilies of retrotransposons, indicating either a differential susceptibility to RIP by different types of TEs, or introgression of already RIPed sequences from a sexual organism [2].

To further investigate the nature and extent of genetic diversity in $V$. dahliae, we have undertaken an analysis of TEs in this species. We report here the identification and characterization of Class I and II transposons in $V$. dahliae, an analysis of their distribution in $V$. dahliae and V. albo-atrum, and discuss their potential role(s) as generators of diversity in the Verticillium genomes.

\section{Results}

\section{Transposable elements in Verticillium dahliae genome} (strain VdLs.17)

Using a combination of bioinformatics predictions and manual inspections, we identified 56 complete Class I retrotransposons and 34 Class II "cut-and-paste" DNA transposons in the VdLs.17 genome. Based on comparison to consensus sequence structures [8], the retrotransposons were identified as members of the LTR class Copia and Gypsy superfamilies, and LINE superfamily I, and the DNA TEs as subclass I members of the TIR superfamilies Tc1/mariner, Activator (hAT) and Mutator (MULE) (Figure 1, Table 1).

\section{LTR retrotransposons of superfamily Copia}

We identified 26 full-length Copia-like TEs, referred to as VdLTRE 1 and 5, respectively (Figure 1A, Table 1). The 24 copies of VdLTRE1 were $5,873 \mathrm{bp}$ in length, flanked by 213 bp LTRs, and contained a single ORF with fused GAG and POL sequences predicted to encode a 1621 amino acid (aa) polyprotein. In contrast, the two copies of VdLTRE5 had shorter (159 bp) LTRs, and GAG and POL ORFs separated by a UGA stop codon. This rarely observed ORF organization requires a leaky stop codon for translation read-through of the POL ORF 3' into the GAG ORF. A conserved CARYYA sequence has been shown to be important for the stop codon readthrough [24], however a search of VdLTRE5 showed that it lacked this canonical sequence motif. A MOTIF search identified in both VdLTRE1 and VdLTRE5 an integrase core domain located $\mathrm{N}$-terminally to a reverse transcriptase domain, placing both elements as members of the Ty1/Copia family of retroelements [25]. These retrotransposases shared the highest sequences similarities with hypothetical proteins of the fungal animal pathogen Penicillium marneffei. Finally, a genome-wide search was conducted that identified two VdLTRE1 solo-LTRs. Such sequences are commonly observed remnants of
LTR retrotransposons, and likely arise from recombination between the direct repeat sequences that flank retroelements [26].

\section{LTR retrotransposons of superfamily Gypsy}

Three families of Gypsy-like elements were discovered and referred to as VdLTRE2, VdLTRE3, and VdLTRE4 (hereafter indicated as VdLTRE2/3/4), and corresponded to VDf35 and VDf90, previously identified as $V$. dahliae retrotransposon-like sequences [4]. These elements were $\sim 6.8$ to $6.9 \mathrm{~Kb}$ in size, $82.5 \%$ identical at the nucleotide level, encoded nearly identical GAG and POL proteins, and possessed LTRs of 476, 433 and $455 \mathrm{bp}$, respectively (Figure $1 \mathrm{~A}$, Table 1 ). There were 22 fulllength elements and 14 solo-LTR sequences in the VdLs.17 genome. The GAG proteins of these Gypsy-like elements contained both gag and zinc finger conserved domains. The $P O L$ ORFs were in a -1 frameshift orientation relative to that of the GAG ORF, and comprised aspartate protease (PR), reverse transcriptase (RT), $\mathrm{RNaseH}$, chromodomain, and integrase (IN) domains in an organization typical of Ty3/Gypsy-like elements $[8,27]$. The top hit from a BLASTP search with the 1129 aa VdLTRE2/3/4 POL predicted proteins was the Cgret LTR element from the closely related cranberry fruit rot fungus Colletotrichum gloeosporioides [28].

\section{Non-LTR retrotransposons of superfamily I}

A LINE-like non-LTR retrotransposon, VdLINE1, was identified as present in 8 copies. The 4,905 bp elements contained separate GAG-like and POL ORFs, and an NCBI conserved domain search identified endonucleaseexonuclease-phosphatase (EEP) and RT domains, as well as a $\mathrm{C}$-terminal $\mathrm{RNaseH}$ domain that defines superfamily I elements [8]. The top hit from a BLASTP search of the predicted 1245 aa POL protein was that of a putative reverse transcriptase of $P$. marneffei (Figure 1A; Table 1).

\section{"Cut-and paste" transposons of superfamily Tc1/mariner} Among the DNA transposons found in the VdLs.17 genome, twenty seven full-length Tc1/mariner-like elements were identified by having their short, perfect inverted terminal repeats (TIRs) flanked by dinucleotide TA target site duplications (TSDs), and the presence between the TIRs of a single intronless ORF encoding a transposase characterized by the conserved endonuclease domain DDE_1 [18] and the N-terminal DNA binding domains HTH_psq and HTH_Tnp_Tc5 (Figure 1B, Table 1, aa sequences of the conserved domains in Additional file 1; Figure S1). On the basis of TIR, ORF and whole TE length and sequence identity, we distinguished three VdLs.17 Tc1/mariner families referred hereafter as DAHLIAE 1, 2 and 3. 


\section{A Ty1/Copia}

Ty3/Gypsy

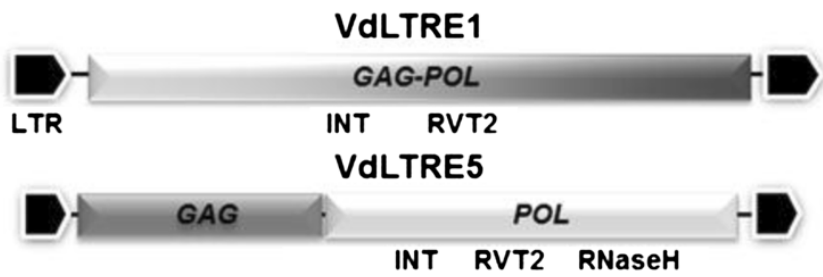

VdLTRE2/3/4
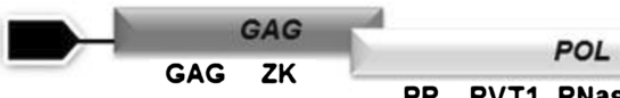

PR RVT1 RNaseH INT CR

LINE-like

VdLINE

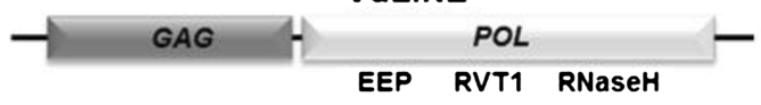

B

\section{Tc1/mariner}

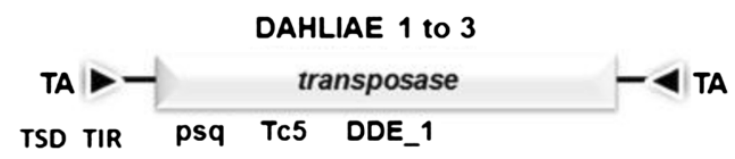

Activator

VdHAT

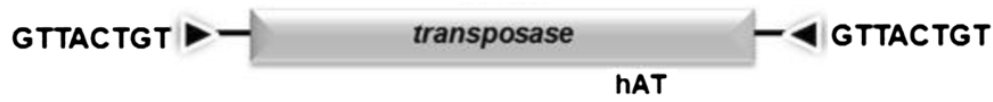

Mutator

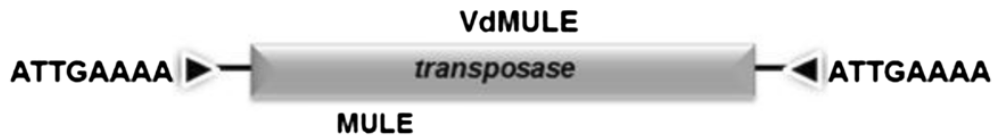

Figure 1 Structure and organization of Class I and II elements identified in the genome of Verticillium dahliae (strain VdLs.17). A) Class I LTR retrotransposons. Schematic representations of the structure of the Ty1/Copia-like and Ty3/Gypsy-like LTR elements, as well as a LINE-like non-LTR retrotransposon. The conserved domains identified in the putative protein sequences encoded by the GAG and POL genes are indicated below the open reading frames (ORFs). VdLTRE1 (upper schematic) contains direct long terminal repeats (LTR), and a fused GAG and POL ORF, while VdLTRE5 GAG and POL ORFs are separated by a UGA stop codon. The highly similar sequences of VdLTRE2, VdLTRE3, and VdLTRE4 (VdLTRE2/3/4) have overlapping GAG and POL ORFs. VdLINE sequence contains linked, but separate, GAG and POL ORFs. Domain abbreviations: INT, integrase (pfam00665); RVT2, reverse transcriptase 2 (pfam07727); RnaseH (pfam0075.16), GAG, group specific antigen; ZK, zinc knuckle (pfam00098); PR, Retropepsin protease (CD00303); RVT_1, reverse transcriptase 1; CR, chromatin organization modifier (CD00024); EEP, endonuclease-exonuclease-phosphatase; B) Class II transposable elements of the superfamilies Tc1/mariner, Activator and Mutator. Each TE type possesses terminal inverted repeats (TIRs) of different length flanking a transposase gene (shown is the corresponding ORF). The Tc1/mariner transposases are characterized by the endonuclease superfamily motif DDE_1 (pfam03184) and the presence of the additional N-terminal DNA-binding domains helix-turn-helix_pipsqueak (here indicated as psq, pfam05225), and helix-turn-helix_Tnp_Tc5 (here indicated as Tc5, pfam3221). The relative position of the hAT dimerization (pfam05699) and the MULE (pfam10551) domains characterizing the Activator and Mutator transposases are also shown. Flanking the TIRs are the nucleotide sequences of the direct target site duplications (TSDs) generated in the fungal genome by the TE insertion.

Among the DAHLIAE1 sequences, we found 25 complete elements about $1.9 \mathrm{~kb}$ in length. These sequences were associated with 41 to 62 bp TIRs that showed conservation of the first four nucleotides ACGT-, and contained 15 to $17 \mathrm{bp}$ internal direct repeats (DRs)
(Additional file 2: Figure S2). We also detected about 10 additional defective DAHLIAE1-like elements, five of which lack both TIRs (not shown). All DAHLIAE1 predicted ORFs started at TE nucleotide position 100 and encoded 551 aa proteins with the DDE_1 endonuclease 
Table 1 Verticillium dahliae (VdLs.17) Class I and II transposable elements identified in this study

\begin{tabular}{|c|c|c|c|c|c|c|c|c|c|}
\hline Name & Family & Length (bp) & LTR (bp) & TIR (bp) & TSD (bp) & Copy number ${ }^{\mathrm{a}}$ & Element domain organization $^{\mathbf{b}}$ & Best BLAST hit $^{\circ}$ & GenBank assession \\
\hline \multicolumn{10}{|l|}{ Class I } \\
\hline VdLTRE1 & Copia & 5873 & 213 & - & - & 24 & $\begin{array}{l}\text { INT (3.53e-14); } \\
\text { RVT_2 (6.12e-55); } \\
\text { RH_Ty1 (1.96e-40) }\end{array}$ & $\begin{array}{l}\text { EEA22357, } \\
\text { Penicilium mameffei, } \\
\text { identities 429/1277 (34\%) }\end{array}$ & HM852745 \\
\hline VdLTRE2 & Gypsy & 6935 & 476 & - & - & 14 & $\begin{array}{l}\text { GAG (2.3e-10); } \\
\text { CCHC (3.36e-06); } \\
\text { PR (9.23e-20): } \\
\text { RVT_1 (2.93e-26); } \\
\text { RH_Ty3 (3.09e-51): }\end{array}$ & $\begin{array}{l}\text { AAG24792 (Cgnet), } \\
\text { Collectotrichum gloeosporiodes, } \\
\text { identities } 673 / 1149(59 \%)\end{array}$ & HM852746 \\
\hline VdLTRE3 & Gypsy & 6828 & 433 & - & - & 4 & see VdLTRE2 & $\begin{array}{l}\text { AAG24792 (Cgnet), } \\
\text { Collectotrichum gloeosporiodes, } \\
\text { identities } 661 / 1101 \text { (61\%) }\end{array}$ & HM852747 \\
\hline VdLTRE4 & Gypsy & 6870 & 455 & - & - & 4 & see VdLTRE2 & $\begin{array}{l}\text { AAG24792 (Cgnet), } \\
\text { Collectotrichum gloeosporiodes, } \\
\text { identities } 661 / 1106 \text { (61\%) }\end{array}$ & HM852748 \\
\hline VdLTRE5 & Copia & 5555 & 159 & - & - & 2 & $\begin{array}{l}\text { INT (1.01e-06); } \\
\text { RVT_2 (8.41e-60); } \\
\text { RH_Ty1 (6.26e-37) }\end{array}$ & $\begin{array}{l}\text { EEA24286, } \\
\text { Penicilium mameffei, } \\
\text { identities 621/1306 (48\%) }\end{array}$ & HM852749 \\
\hline VdLINE1 & LINE & 4905 & - & - & - & 8 & $\begin{array}{l}\text { APE }(5.68 \mathrm{e}-10) ; \\
\text { RVT_1 (3.59e-20); } \\
\text { RH (7.53e-09) }\end{array}$ & $\begin{array}{l}\text { EEA18490, } \\
\text { Penicilium mameffei, } \\
\text { identities 879/1269 (70\%) }\end{array}$ & HM852750 \\
\hline \multicolumn{10}{|l|}{ Class II } \\
\hline DAHLIAE1a & Tc1/mariner & 1861 & - & 45 & 2 & 12 & $\begin{array}{l}\text { HTH_psq (3.16e-06); } \\
\text { HTH_Tnp_Tc5 (7.75e-04); } \\
\text { DDE_1 (1.35e-58) }\end{array}$ & $\begin{array}{l}\text { EGU83431, } \\
\text { Fusarium oxysporum, } \\
\text { identities 317/551 (58\%) }\end{array}$ & JN160806 \\
\hline DAHLIAE1b & Tc1/mariner & 1862 & - & 41 & 2 & 4 & $\begin{array}{l}\text { HTH_Tnp_Tc5 (4.32e-03); } \\
\text { DDE_1 (6.10e-58) }\end{array}$ & $\begin{array}{l}\text { EGU83431, } \\
\text { Fusarium oxysporum, } \\
\text { identities 317/539 (59\%) }\end{array}$ & JN160807 \\
\hline DAHLIAE1C & Tc1/mariner & 1862 & - & 55 & 2 & 1 & $\begin{array}{l}\text { HTH_psq (1.37e-06); } \\
\text { DDE_1 (1.72e-59) }\end{array}$ & $\begin{array}{l}\text { EGU83431, } \\
\text { Fusarium oxysporum, } \\
\text { identities 322/546 (59\%) }\end{array}$ & JN160816 \\
\hline DAHLIAE1d & Tc1/mariner & 1862 & - & 53 & 2 & 9 & $\begin{array}{l}\text { HTH_psq (1.37e-07); } \\
\text { DDE_1 (1.72e-58) }\end{array}$ & $\begin{array}{l}\text { EGU83431, } \\
\text { Fusarium oxysporum, } \\
\text { identities 317/548 (58\%) }\end{array}$ & JN160808 \\
\hline DAHLIAE1e & Tc1/mariner & 1861 & - & 62 & 2 & 1 & DDE_1 (2.42e-46) & $\begin{array}{l}\text { EGU83431, } \\
\text { Fusarium oxysporum, } \\
\text { identities 222/363 (61\%) }\end{array}$ & JN160817 \\
\hline DAHLIAE2 & Tc1/mariner & 1909 & - & 62 & 2 & 1 & $\begin{array}{l}\text { HTH_psq (3.31e-08); } \\
\text { HTH_Tnp_Tc5 (2.12e-12); } \\
\text { DDE_1 (2.95e-68) }\end{array}$ & $\begin{array}{l}\text { ABG26270 (OPHIO2), } \\
\text { Ophiostoma ulmi, } \\
\text { identities } 275 / 529 \text { (52\%) }\end{array}$ & JN160809 \\
\hline
\end{tabular}


Table 1 Verticillium dahliae (VdLs.17) Class I and II transposable elements identified in this study (Continued)

\begin{tabular}{|c|c|c|c|c|c|c|c|c|c|}
\hline DAHLIAE3 & Tc1/mariner & 1949 & - & 102 & 2 & 1 & $\begin{array}{l}\text { HTH_Tnp_Tc5 (1.36e-06); } \\
\text { DDE_1 (5.53e-28) }\end{array}$ & $\begin{array}{l}\text { EED116641, } \\
\text { Talaromyces stipitatus, } \\
\text { identities 148/400 (37\%) }\end{array}$ & JN160810 \\
\hline VdHAT1 & Activator & 3164 & - & 22 & 8 & 1 & $\begin{array}{l}\text { Dimer_Tnp_hAT } \\
\text { (7.15e-19) }\end{array}$ & $\begin{array}{l}\text { EAQ93808, } \\
\text { Chaetomium globosum, } \\
\text { identities 361/814 (44\%) }\end{array}$ & JN160811 \\
\hline VdHAT3 & Activator & 2809 & - & 20 & - & 1 & $\begin{array}{l}\text { Dimer_Tnp_hAT } \\
(1.54 \mathrm{e}-14)\end{array}$ & $\begin{array}{l}\text { EED11981, Talaromyces stipitatus, } \\
\text { identities 265/652 (40\%) }\end{array}$ & JN160812 \\
\hline VdMULE1 & Mutator & 3613 & - & 86 & 8 & 1 & MULE (3.86e-17) & $\begin{array}{l}\text { EFZ03845, Metarhizium anisopliae, } \\
\text { identities 385/569 (68\%) }\end{array}$ & JN160813 \\
\hline VdMULE2 & Mutator & 3538 & - & 84 & - & 1 & MULE (3.49e-21) & $\begin{array}{l}\text { EFZ03845, Metarhizium anisopliae, } \\
\text { identities 306/441 (69\%) }\end{array}$ & JN160814 \\
\hline VdMULE3 & Mutator & 3406 & - & 73 & - & 1 & MULE (3.61e-20) & $\begin{array}{l}\text { EFZ03845, Metarhizium anisopliae, } \\
\text { identities 372/548 (68\%) }\end{array}$ & JN160815 \\
\hline
\end{tabular}




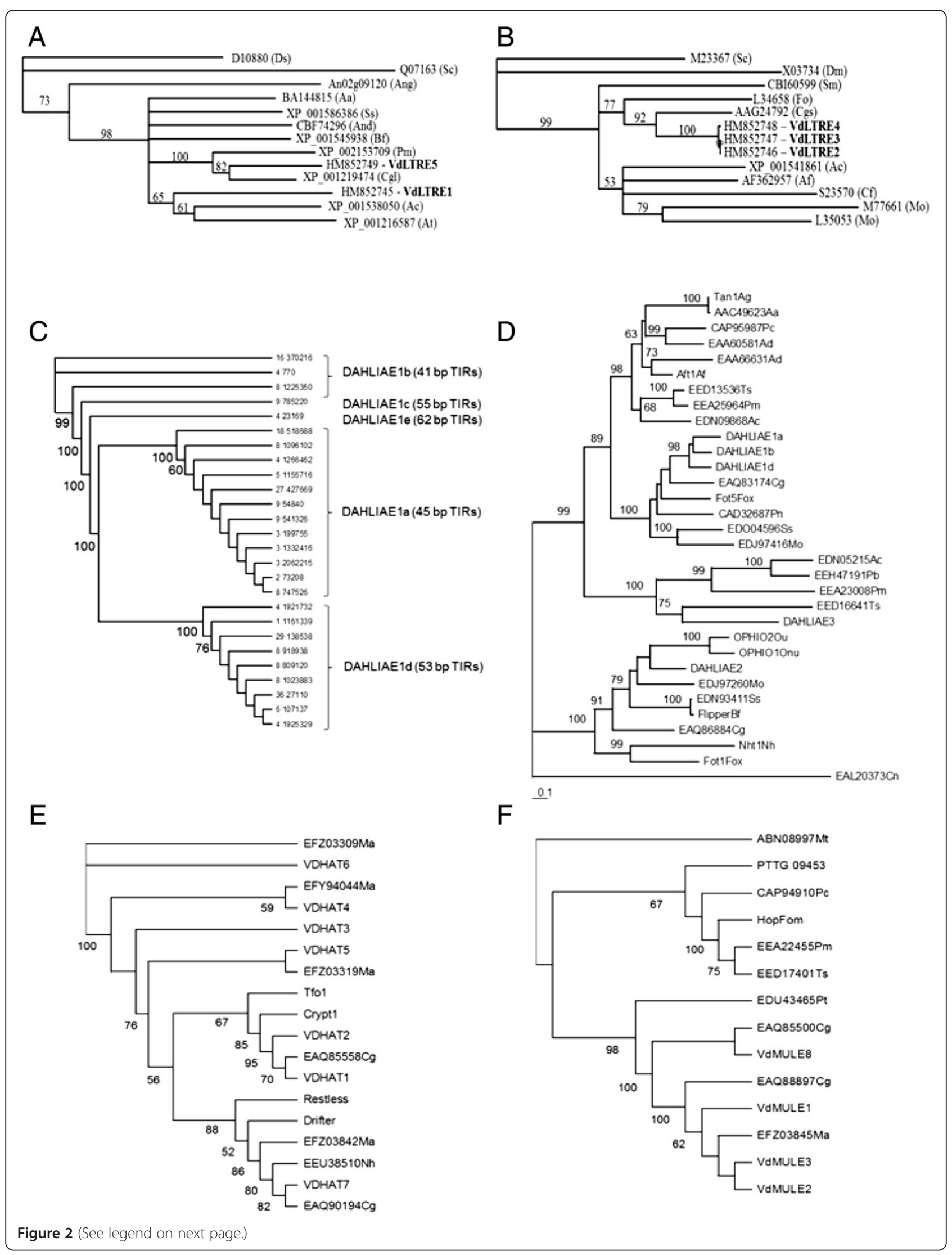


(See figure on previous page.)

Figure 2 Phylogenetic analysis of VdLs.17 Class I and II elements. A) Ty1/Copia LTR retrotransposons, analysis of the reverse transcriptases (amino acid sequences); B) Ty3/Gypsy LTR retrotransposons, analysis of the reverse transcriptases (amino acid sequences); C) Tc1/mariner-like DAHLAIE 1, analysis of the full-length elements (TIR-comprising nucleotide sequences). Each element is identified by its chromosome location (supercontig and nucleotide position); D) Tc1/mariner-like DAHLAIE 1 to 3, analysis of complete transposases (amino acid sequences);

E) Activator-like VdHAT elements, analysis of hAT transposase domains (amino acid sequences); F) Mutator-like VdMULE elements, analysis of MULE transposase domains (amino acid sequences). The trees indicate the GenBank accession number of the amino acid sequences analyzed, along with the abbreviation of the scientific names of organisms of origin. Abbreviations are as follow: Aa, Alternaria alternata (A), Aspergillus awamori (D); Ac, Ajellomyces capsulatus; Af, Aspergillus flavus (A), Aspergillus fumigatus (D); Ad/And, Aspergillus nidulans (A), Ag/Ang, Aspergillus niger (D); At, Aspergillus terreus; Bf, Botryotinia fuckeliana; Cn, Cryptococcus neoformans; Cgl/Cg, Chaetomium globosum; Cf, Cladospororium fulvum; Cgs, Colletotrichum gloeosporioides; Dm, Drosophila melanogaster; Ds, Drosophila simulans; Fo/Fox, Fusarium oxysporum; Fom, Fusarium oxysporum melonis; Ma, Metharhizium anisopliae; Mo, Magnaporthe oryzae; Mt, Medicago truncatula; Pc, Penicillium crysogenum; Pm, Penicillium marneffei; Pn, Phaesphaeria nodorum; Pt, Pyrenophora tritici-repentis; Sc, Saccharomyces cerevisiae; Ss, Sclerotinia sclerotiorum; Sm, Sordaria macrospora; Ts, Talaromyces stipitatus. Scale bar corresponds to 0.1 substitutions per amino acid or nucleotide.

domain spanning aa positions 154-364 and one or both the N-terminal DNA-binding domains HTH_psq and HTH_Tnp_Tc5 at aa positions 7-50 and 57-120, respectively (Figure 1B, Table 1). BLASTP with DAHLIAE1 proteins revealed greatest sequence similarities $(58-61 \%$ aa identity) with sequences from ascomycete fungi, in particular, with a predicted protein from the phytopathogen F. oxysporum (strain Fo5176) (Table 1).

To establish the relationships among the DAHLIAE1 transposons, we performed phylogenetic analysis using the full-length nucleotide sequences, which resulted in the classification of the 5 subfamilies DAHLIAE1 a to e (Table 1, Figure 2C). Members of the same subfamily shared the same TIR length and were 95-100\% identical to each other. Sequence homologies between members of the different subfamilies ranged from 73 to $74 \%$. DAHLIAE $1 \mathrm{a}$ and $1 \mathrm{~d}$, with 12 and 9 complete copies each, respectively, were the largest subfamilies and the most abundant DNA transposons in VdLs.17 genome. DAHLIAE1 $c$ and $e$ were each present in only a single complete copy, and encoded what are likely to be nonfunctional transposases due to in-frame early stop codons.

Only one complete copy of DAHLIAE2 was detected in VdLs.17. The element was 1,909 bp long with 62 bp TIRs, and harbored an ORF coding for a putative 542 aa polypeptide (Table 1). The TIRs, like in DAHLIAE1, started with the nucleotides ACGT-, comprised three mismatches, and the DR sequence TTTCGGACACCCCCCCC- repeated three times at the 5'-end of the TEs (Additional file 2: Figure S2). The DAHLIAE2 ORF started at nucleotide position 187, and the encoded transposase shared the greatest overall sequence homology $(52 \%$ aa identity) with OPHIO2 from the causal agent of Dutch elm disease Ophiostoma ulmi [29].

Like DAHLIAE2, only one full-length copy of DAHLIAE3 was present in VdLs.17. The consensus sequence was 1,949 bp long, with 102-bp TIRs. The 5' TIR started with the sequence CCCGT- and TIR alignment revealed one mismatch, and lack of internal direct duplications or palindromes (not shown). DAHLAIE3 contained a 1,653-bp ORF starting at the TE nucleotide position 144, and encoded a 550 aa transposase with similarities to a putative 427 aa transposase from the fungal human pathogen Talaromyces stipitatus (Table 1).

\section{"Cut-and paste" transposons of superfamily Activator}

Seven VdLs.17 sequences, designated VdHAT 1 to 7, were found to putatively encode 55 to 85 aa hAT dimerization domains, which characterize the carboxy termini of the transposases of Activator-like element transposases [30] (domain aa sequences shown in Additional file 3: Figure S3A). We were able to define the consensus sequence and flanking short imperfect TIRs of two single copy elements, VdHAT 1 and 3, and identify for VdHAT1 the 8-bp TSD GTTACTGT containing a typical central TA-dinucleotide. The complete VdHAT 1 and 3 elements were 3,164 and 2,809 bp in length, with 22 and 20 bp TIRs, respectively (Figure 1B, Table 1). The TIRs of each element comprised one mismatch and, while the VdHAT3 TIRs started with the sequence ATTTG-, VdHAT1 TIRs were characterized by the motif CAGNG- (Additional file 2: Figure S2), which is also present in other fungal hAT-like elements such as the Cryphonectria parasitica Crypt1 [31], the F. oxysporum Drifter and Tfo1 [32,33], and the Tolypocladium inflatum Restless [34]. Analysis of the VdHAT partial and complete nucleotide sequences using BLASTN and CLUSTALW revealed no significant regions of similarity to Activator-like sequences from other organisms, suggesting that each VdHAT was VdLs.17-specific. ORF Finder searches with VdHAT TEs and the analysis of corresponding ESTs supported the presence of a single transposase-coding gene. However, the presence in most of the VdHATs of in-frame early stop codons suggested that partial, likely non-functional proteins would be generated. BLASTX analysis showed that while the best hits for VdHAT 1 and 2 were two sequences from C. globosum, the best matches for VDHAT 3 to 7 sequences 

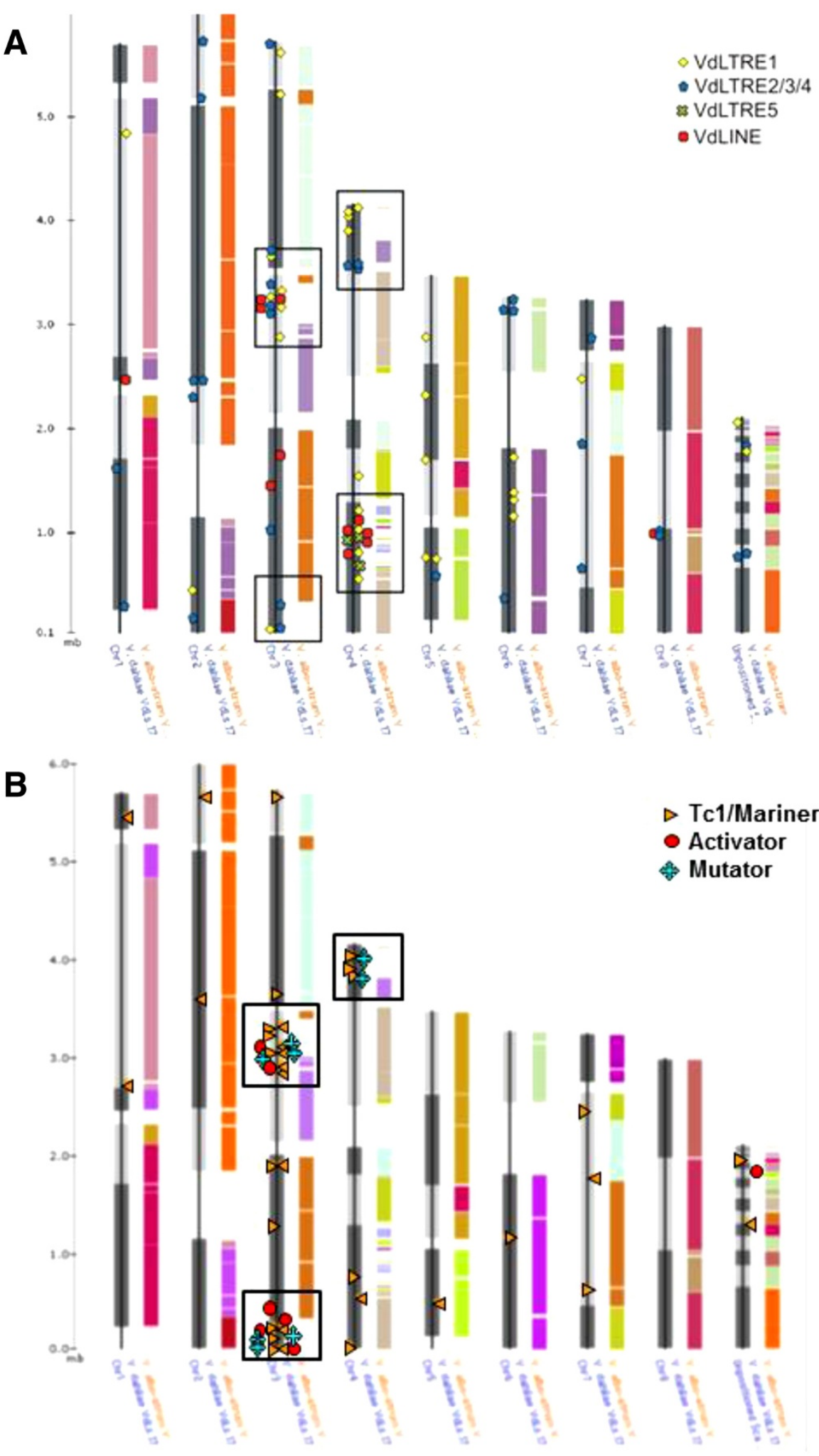

Figure 3 Distribution of Class I and II TEs within the VdLs.17 genome. The ChromoMap genome browser available online at the BROAD Institute website was used to visualize the TE sequences against a synteny map of the VdLs.17 and VaMs.102 sequenced genomes. The biased chromosome localization of the retroelements (A) and the DNA elements (B) in the "lineage-specific" regions of Vd.Ls17 are outlined in black. 

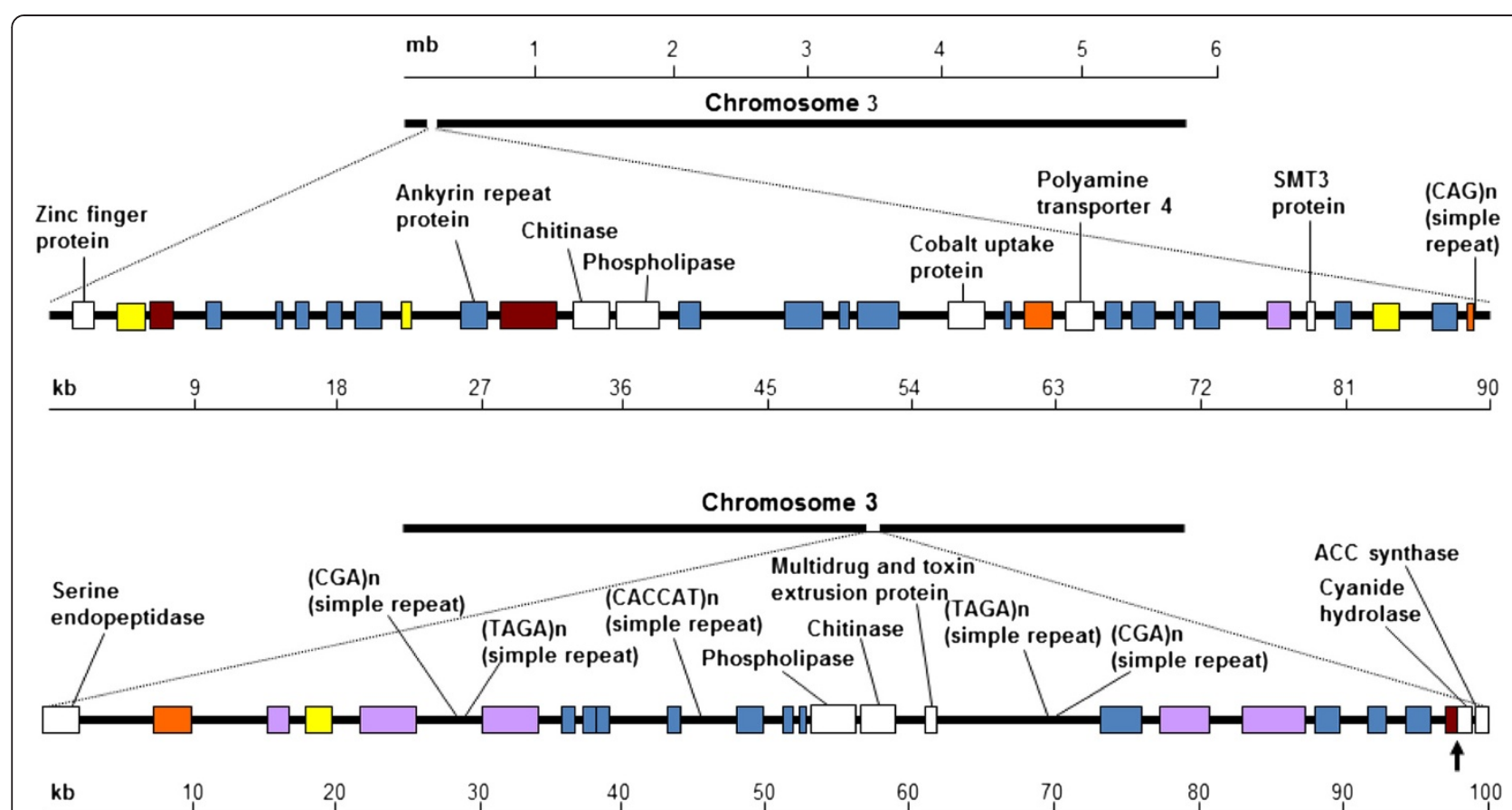

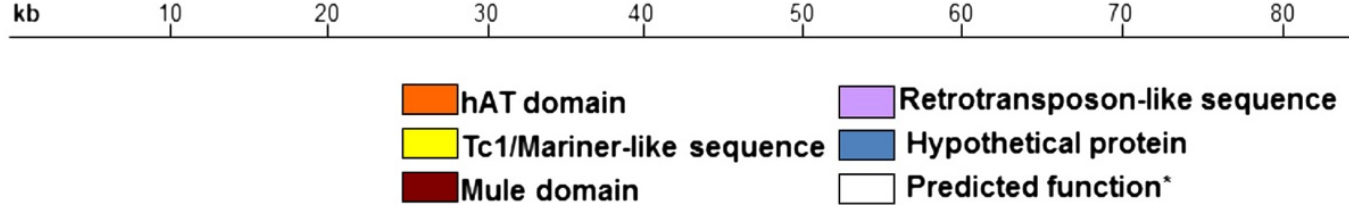

Figure 4 Genetic context of Class I and II transposable elements in VdLs.17. The sequence segments include $90 \mathrm{~kb}$ from supercontig 4 nucleotide positions 162,000-222,000 (top), and $100 \mathrm{~kb}$ from supercontig 8 nucleotide positions 1006000 - 1106000 (bottom), of chromosome 3. The arrow indicates the position of the putative cyanide hydratase fusion protein (VDAG_04885) containing both nitrilase and MULE transposase functional domains (pfam 00795 and 10551, respectively). *Predicted gene names were assigned by the Broad Institute (www.broadinstitute.org/annotation/genome/verticillium_dahliae).

were to four different predicted proteins of the recently sequenced entomopathogenic ascomycete Metarhizium anisopliae [35] (Table 1, Additional file 4: Table S1).

\section{"Cut-and paste" transposons of superfamily Mutator} BLASTP search using the 94 aa MULE domain from the active Mutator-like element Hop1 of $F$. oxysporum f. sp. melonis [36] revealed the presence of eight predicted MULE proteins in VdLs.17 (domain aa sequences shown in Additional file 3: Figure S3B). Manual inspection of the corresponding genomic sequences, which we refer to as VdMULE 1 to 8 , resulted in the discovery of three potentially complete elements VdMULE 1 (3.6 Kb), 2 (3.5 Kb) and 3 (3.4 $\mathrm{Kb})$, each encoding a single ORF, and containing 86,82 , and 53 bp TIRs, respectively. An 8 bp TSD (ATTGAAAA) was associated with one element only, and defective elements ranged in size from 363 to 2,371 bp (Table 1, Additional file 4: Table S1).

Alignment of the VdMULE nucleotide sequences with Hop1 TIRs showed the presence of the terminal consensus GGNAA, and of sub-terminal DRs of different lengths. In particular, while VdMULE 1 and 2 had three 15-bp and 17-bp repeats, respectively, VdMULE3, like Hop1, possessed 5 and 6 bp duplications (Additional file 2: Figure S2). Among the VdMULEs, the highest nucleotide sequence homology was observed between the three full-length elements (55\% to $69 \%$ identity). VdMULE8 appeared to be the most divergent, with no significant similarity to any other VdMULE element.

VdMULE elements were predicted to harbor single transposase genes with different exon/intron structures. In particular, we found a single exon in the VdMULE1, 2, 3 and 6 genes, four exons in VdMULE4, two exons in VdMULE5 and five exons in the VdMULE8 genes, respectively. Complete MULE domains (94-95 aa) were present only in transposases of the full-length elements. The remaining transposases were characterized by $\mathrm{N}$ - or C-terminal domain truncations of 21 to 57 aa. Notably, the predicted 489 aa VdMULE7 protein (VDAG_04885) was found to be the in-frame product of the fusion between a nitrilase and a MULE-transposase coding sequence. The hypothetical protein harbors a 177 aa Nterminal nitrilase domain (pfam00795; involved in nitrile 
hydrolysis to ammonia and carboxyl acid) spanning aa positions 3-180, and a truncated 74 aa MULE domain spanning aa positions 363-436. VdMULE8 transposase (VDAG_04851) architecture was also characterized by the presence of a 20 aa zinc-knuckle (zf-CCHC, pfam00098) at its carboxy terminus. Proteins with the highest sequence homology to the VdMULE transposases, which ranged from $55 \%$ to $79 \%$ identity, were from C. globosum and M. anisopliae (Table 1).

\section{Distribution of Class I and II TEs in the VdLS.17 genome}

An in-depth analysis of transposon distribution in VdLs.17 genome revealed that the most abundant elements, VdLTREs $1-4$ and Tc1/mariner DAHLIAE1, were dispersed throughout the genome's eight chromosomes, and in unpositioned scaffold sequences. However, there was notable TE clustering in chromosomes 3 and 4 (Figure $3 \mathrm{~A}$ and $\mathrm{B}$ ). In particular, supercontings 4 and 8 of chromosome 3 appeared to contain "hot-spots" of TE insertions, harboring $21 \%$ and $20 \%$ of the transposons, respectively. These supercontigs also contain VdLs.17 LS

Table 2 Survey of VdLs.17 ESTs corresponding to the transposons identified in this study

\begin{tabular}{|c|c|c|c|}
\hline \multirow[t]{2}{*}{ Transposon } & \multicolumn{3}{|c|}{ Number of ESTs ${ }^{a}$} \\
\hline & CM & $\mathrm{CM}+\mathrm{RE}$ & $\mathrm{CM}-\mathrm{N}$ \\
\hline \multicolumn{4}{|l|}{ Class I } \\
\hline VdITRE1 & 43 & 21 & 23 \\
\hline VdITRE2 & 31 & 26 & 33 \\
\hline VdITRE3 & $24^{* *}$ & 16 & $21^{* * *}$ \\
\hline VdITRE4 & $25^{* *}$ & $23^{*}$ & 28 \\
\hline VdITRE5 & 3 & 2 & 5 \\
\hline VdLINE1 & 13 & 11 & 4 \\
\hline \multicolumn{4}{|l|}{ Class II } \\
\hline DAHLIAE1a & 4 & - & 6 \\
\hline DAHLIAE1b & - & 4 & 4 \\
\hline DAHLIAE1d & 3 & - & 11 \\
\hline DAHLIAE1e & 6 & - & 3 \\
\hline DAHLIAE2 & 6 & 2 & 5 \\
\hline DAHLIAE3 & - & 4 & - \\
\hline VdHAT1 & 12 & 2 & 8 \\
\hline VdHAT2 & - & 1 & - \\
\hline VdHAT3 & - & 1 & - \\
\hline VdMULE2 & 4 & 2 & - \\
\hline VdMULE3 & - & 5 & - \\
\hline VdMULE8 & - & 2 & - \\
\hline \multicolumn{4}{|c|}{$\begin{array}{l}\text { VdLs.17 expressed sequence tag (EST) collections were generated from } \\
\text { cultures grown in CM (complete medium), CM + RE (CM amended with root } \\
\text { extract), or CM-N (CM lacking nitrogen source). For VdLTRE2, VdLTRE3 and } \\
\text { VdLTRE4, the corresponding ESTs aligned with all three elements except that } \\
\text { * one EST aligned with only VdLTRE4, }{ }^{* *} \text { four ESTs aligned with both VdLTRE } 3 \\
\text { and 4, and *** one EST aligned with only VdLTRE3. }\end{array}$} \\
\hline
\end{tabular}

(lineage-specific) regions 1 and 2, which are absent from the VaMs.102 genome [2]. The non-random TE distribution of the DDE_1, hAT and MULE motifs was further assessed, and odds ratio analyses [37] did indeed show that the LS regions are significantly enriched in transposon motifs $(P<0.05)$ relative to that of the total predicted gene distribution in the genome assembly of VdLs.17 (Additional file 5: Table S2). One approximately $100 \mathrm{~Kb}$ region from each of LS regions 1 and 2 was selected to illustrate the genetic context of some TEs (Figure 4). This figure highlights how the transposons are present in gene-rich locations, and shows a putative sequence duplication event involving genes encoding a chitinase and a phospholipase, in addition to those previously described [2].

\section{The Verticillium albo-atrum VaMs.102 genome contains few transposons}

While TEs were prevalent in the $V$. dahliae VdLs.17 genome, few repetitive sequences were identified in VaMs.102. BLASTN and BLASTX queries against the $V$. albo-atrum genome using VdLs.17 Class I and II transposons identified one intact, full-length element having 99.9\% sequence identity to VdLTRE1, one full-length but degenerate Copia-like element, and one partial sequence of approximately $1 \mathrm{~kb}$, also of the Copia-type. Only short (45 to $263 \mathrm{bp}$ ) sequences were found that had 80 to 91 percent identity to the Gypsy-like elements. Lastly, no sequences corresponding to VdLTRE5 were identified, and remarkably, neither full-length nor incomplete Class II TEs were detected in the VaMs.102 genome.

\section{VdLs.17 Class I and II transposons are transcriptionally active}

To assess potential transcriptional activity, we inspected the three VdLs.17 EST libraries generated in support of the Verticillium genome sequencing project [2] in search of sequences corresponding to the different TE types described herein. These libraries were obtained from fungal cultures grown on control complete medium (CM; 10,000 ESTs), CM supplemented with root extract from the host plant lettuce $(\sim 5,000 \mathrm{ESTs})$, or CM lacking nitrogen $(\sim 5,000 \mathrm{ESTs})$ [2]. We identified ESTs matching all of the Class I and II transposons in at least one of the growth conditions (Table 2).

The EST analysis showed that Class I elements were transcriptionally active under all of the growth conditions. Although similar levels of expression were observed for the $\mathrm{CM}+$ root extract and $\mathrm{CM}$-nitrogen cultures, numbers of VdLTRE1 ESTs were substantially higher than those of all other elements. In contrast, within each DNA TE family, a differential level of expression was observed according to the fungal growth condition. For instance, while DAHLIAE1 a, d and e 
Table 3 Survey of Verticillium dahliae and V. albo-atrum isolates for the presence of Class I and II transposons identified in VdLs.17 genome

\begin{tabular}{|c|c|c|c|c|c|}
\hline Isolate & Geographic Origin & Host & VCG $^{\mathbf{b}}$ & Retrotransposons & "Cut and Paste" DNA transposons \\
\hline \multicolumn{6}{|l|}{ V.dahliae } \\
\hline VdLs. $17^{\mathrm{a}}$ & CA & Lettuce & $2 \mathrm{~B}$ & Copia (VdLTRE 1 and 5), Gypsy (VdLTRE2/3/4) & $\begin{array}{l}\text { Tc1/mariner (DAHLIAE } 1 \text { to 3), } \\
\text { Activator (VdHAT } 1 \text { to } 7 \text { ), } \\
\text { Mutator (VdMULE } 1 \text { to } 8 \text { ) }\end{array}$ \\
\hline VdT9 & CA & Cotton & 1 & Copia (VdLTRE 1 and 5), Gypsy (VdLTRE2/3/4) & - \\
\hline VdV44 & TX & Cotton & 1 & Copia (VdLTRE 1 and 5), Gypsy (VdLTRE2/3/4) & - \\
\hline VdPCW & CA & Pepper & 3 & Copia (VdLTRE 1 and 5), Gypsy (VdLTRE2/3/4) & Tc1/mariner (DAHLIAE1d) \\
\hline Vd70.21 & unknown & unknown & 3 & Copia (VdLTRE 1 and 5), Gypsy (VdLTRE2/3/4) & nd \\
\hline VdDvd-T5 & ON & Tomato & $2 \mathrm{~A}$ & Copia (VdLTRE1), Gypsy (VdLTRE2/3/4) & $\begin{array}{l}\text { Tc1/mariner (DAHLIAE1d), } \\
\text { Activator (VdHAT1) }\end{array}$ \\
\hline$V d B B$ & ID & Potato & $4 \mathrm{~A}$ & Gypsy (VdLTRE2/3/4) & $\begin{array}{l}\text { Tc1/mariner (DAHLIAE2), } \\
\text { Activator (VdHAT2) }\end{array}$ \\
\hline VdWM & TX & Cotton & $2 \mathrm{~A}$ & Gypsy (VdLTRE2/3/4) & nd \\
\hline $\mathrm{VdPH}$ & CA & Pistachio & $2 \mathrm{~A}$ & Copia (VdLTRE1), Gypsy (VdLTRE2/3/4) & - \\
\hline Vd115 & Syria & Cotton & $2 \mathrm{~B}$ & Copia (VdLTRE1), Gypsy (VdLTRE2/3/4) & nd \\
\hline VdS39 & $\mathrm{OH}$ & Soil & $4 \mathrm{~B}$ & Copia (VdLTRE1), Gypsy (VdLTRE2/3/4) & Tc1/mariner (DAHLIAE2) \\
\hline VdCW & WA & Cherry & $4 \mathrm{~A} / \mathrm{B}$ & Copia (VdLTRE1), Gypsy (VdLTRE2/3/4) & Tc1/mariner (DAHLIAE2) \\
\hline \multicolumn{6}{|c|}{ V. albo-atrum } \\
\hline VaMs.102 ${ }^{a}$ & PA & Alfalfa & nd & Copia (VdLTRE1) & - \\
\hline Va383-2 & ON & Potato & nd & Copia (VdLTRE1) & Tc1/mariner (DAHLIAE2) \\
\hline Va4ATC & ON & Potato & nd & Copia (VdLTRE1) & Tc1/mariner (DAHLIAE2) \\
\hline VaV5591 & CA & Cauliflower & nd & Copia (VdLTRE1), Gypsy (VdLTRE2/3/4) & - \\
\hline Va462 & $\mathrm{MN}$ & Potato & nd & nd & Tc1/mariner (DAHLIAE2) \\
\hline VaChile1 & Chile & Kiwi & nd & - & Tc1/mariner (DAHLIAE2) \\
\hline VaPSU140 & PA & Ailanthus altissima & nd & - & Tc1/mariner (DAHLIAE2) \\
\hline Va48557 & UK & Tomato & nd & - & Tc1/mariner (DAHLIAE2) \\
\hline VaV481 & UK & Hops & nd & - & Tc1/mariner (DAHLIAE2) \\
\hline VaV104b & $\mathrm{PEI}$ & Potato & nd & - & nd \\
\hline VaV4901 & PEl & Potato & nd & - & nd \\
\hline
\end{tabular}

The presence of the indicated Class I and Class II transposable elements was determined by Southern hybridization or PCR amplification using gene-specific

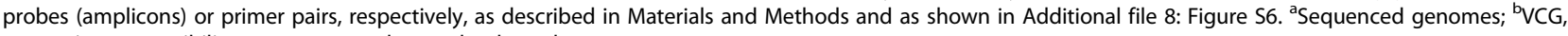
vegetative compatibility groups; -, not detected; nd, no data.

were transcriptionally active primarily in control and nitrogen starvation conditions, ESTs for DAHLIAE3, VdHAT 2 and 3 and VdMULE 3 and 8 were detected only in the library from cultures grown in the root extract-amended medium.

Abiotic stress can stimulate expression of TEs in fungi $[38,39]$. We therefore used reverse transcription-PCR to make a semi-quantitative assessment of the expression of selected VdLs.17 DNA transposases in response to a heat shock treatment $\left(42^{\circ} \mathrm{C}\right.$ for 40 minutes). The resulting data provided evidence that while the expression of DAHLIAE1, DAHLIAE3, VdMULE2, and VdMULE3 was induced at $42^{\circ} \mathrm{C}$, VdMULE8 expression appeared to be unaffected by the treatment, and DAHLIAE2 responded negatively to the treatment (Additional file 6: Figure S4).

\section{Phylogenetic analyses}

To gain a better understanding of the evolutionary history of the different transposable elements identified in VsLs.17, we studied their phylogenetic relationships with homologous sequences from other fungal species. Analyses were done as described in the Methods section and using the aa sequences of the retroelement RT domains, predicted aa sequences of the complete Tc1/mariner proteins, and conserved domains of the VdHAT and VdMULE transposases (Figure 2). 
Consistent with the predictions based on the organization of the POL domains, VdLTRE1 and VdLTRE5 clustered with known fungal Copia-like elements. However, bootstrap support separated the two types of elements into distinct clades, with VdLTRE1 being most closely related to sequences from Aspergillus capsulatus and $A$. terreus (Figure 2A). VdLTREs 2, 3 and 4 clustered together and with known Gypsy elements, showing close relationships with the C. gloeosporioides element Cgret [28] and Skippy from F. oxysporum [40] (Figure 2B).

Although bootstrap values supported a monophyletic origin for all the fungal DDE_1 transposases considered, none of the VdLs.17 DDE_1 transposases clustered with sequences from the basidiomycetes Cryptococcus neoformans (animal pathogen) and Ustilago maydis (plant pathogen), or with sequences from the four different Aspergillus species included in the analysis (Figure 2D). While the three DAHLIAE families, whose members share 28 to $34 \%$ identity, separated into 3 different lineages before speciation, DAHLIAE1 differentiated into subfamilies, sharing 67 to $72 \%$ aa identity, apparently after insertion into the VdLs.17 genome. Both DAHLIAE1- and DAHLIAE2-containing clades also comprised proteins from the Sordariomycetes C. globosum, F. oxysporum, M. oryzae and the Leotiomycete $S$. sclerotiorum, which are closely related to Verticillium spp. However, DAHLIAE 3 grouped with sequences from the more distantly related Erotiomycete species Ajellomyces capsulatus, P. marneffei, Paracoccidioides brasiliensis and T. stipitatus. Among the fungal proteins clustering with DAHLIAE2, all of which possess the HTH_psq/HTH_Tnp_Tc5/DDE_1 domain organization, we found transposases of well characterized plant pathogen TEs such as Flipper of B. fuckeliana [41], Fot1 of $F$. oxysporum [42], Nht1 of Nectria haematococca [43], and OPHIO 1 and 2 of O. nova-ulmi [29].

Sequences with similarities to VdLs.17 hAT and MULE domains were identified in a much more limited number of fungi. Sequence alignments of the VdhAT and VdMULE domains revealed core regions of 53 and 69 aa, respectively, and led to the identification of amino acid positions that are conserved across all fungal species examined (alignments shown in Additional file 3: Figure S3). While the VdHATs were separated into 6 different clades, all VdMULEs clustered together (Figure 2 $E$ and F). Bootstrap values in the range of $50-60 \%$ or below did not support a monophyletic origin for the VdHATs. In the clade containing VdHAT 1 and 2, there were sequences from the Activator-like transposons Crypt1 of the chestnut blight fungus Cryphonectria parasitica [31] and Tfo1 of F. oxysporum [33], together with one sequence from $C$. globosum. VdHAT7 was more closely related to Drifter, a hAT element from $F$. oxysporum [32], and Restless from the cyclosporineproducing fungus $T$. inflatum [34], as well as to sequences from C. globosum, M. anisopliae, and N. haematococca. VdHATs 3 to 6 were grouped with $M$. anisopliae sequences only (Figure 2E). The VdMULE clade comprised $M$. anisopliae and C. globosum sequences and appeared to be unrelated to the clade with the $F$. oxysporum element Hop and sequences from P. crysogenum, P. marneffei, and T. stipitatus (Figure 2F).

\section{Evidence of repeat induced point (RIP) mutation in VdLs.17 TEs}

In a previous study we surveyed VdLs.17 retrotransponsons for evidence of RIP-like mutations and found them only in Gypsy-like sequences [2]. Here we extended the analysis to all VdLs.17 DNA transposons. Although the sequence divergence for the Activator- and Mutator-like elements was significant, RIPCAL searches [44], which included VdLTRE2-like sequences as positive control for RIPing, revealed no clear bias for RIP-like mutations in any of the VdLs.17 "cut-and-paste" elements (Additional file 7: Figure S5).

\section{Distribution of the VdLs.17 Class I and II TEs in other V. dahliae and V. albo-atrum isolates}

The distribution of the Class I and II transposons identified in VdLs.17 was investigated in another $21 \mathrm{~V}$. dahliae and $V$. albo-atrum isolates from various hosts and geographic regions, including the $V$. dahliae vegetative compatibility group (VCG) tester strains described by Joaquim and Rowe [45] (results summarized in Table 3 and shown in Additional file 8: Figure S6). The Tc1/ mariner DAHLIAE2 showed the most widespread distribution among the two species, being present in 33\% and $66 \%$ of the Vd and Va isolates, respectively. The Copia VdLTRE1 and the Gypsy VdLTR2/3/4 were detected in multiple copies in almost all Vd isolates, whereas the Copia-like element VdLTRE5 was found only in VdLs.17 and four other Vd isolates, and always in low copy number (Additional file 8: Figure S6A). Only four Va isolates were conclusively positive for the presence of VdLS.17like retroelements. The Tc1/mariner DAHLIAE1 and Activator-like sequences were, like the retroelements, limited in their distribution, and represented primarily in $V$. dahliae (Additional file 8: Figure S6B). Lastly, Tc1/ mariner DAHLIAE3 and VdMULE elements appeared to be specific to VdLs.17.

\section{Discussion}

We originally set out to gain a better understanding of the nature and extent of genetic diversity in phytopathogenic Verticillium spp. and, to this end identified mobile elements in the sequenced $V$. dahliae and $V$. albo-atrum genomes, and explored their distribution in other strains 
of different origins. Our genome-wide search yielded complete retroelements and "cut-and-paste" DNA transposons, whose structure we characterized in detail. Among the VdLs.17 LTR sequences that we identified, the Ty1/Copia-like VdLTRE5 had the GAG and POL ORFs in an organization that is very rare [46], and uses a leaky stop codon for translation of the POL ORF that is downstream of the GAG ORF. VdLTRE5 did not have, downstream of the GAG stop codon, the conserved CARYYA sequence, which has been previously shown to be important for this stop codon read-through [24]. However, the Ty1/Copia-like element Tca2 of Candida albicans also does not possess this sequence and it has been proposed that the sequences responsible for stop codon read-through of Tca 2 can be multiple, remote, and scattered throughout the element [47]. The same may therefore be true for VdLTRE5.

The VdLs.17 retrotransposons VdLTRE 2, 3, and 4 were identifiable as Gypsy elements in their having GAG and $P O L$ genes with the $P O L$ ORFs in a -1 frameshift orientation relative to that of the GAG ORF [46]. A notable feature differentiating these three TE families, which are predicted to encode almost identical polyproteins, was the difference in their LTR lengths. LTR elements contain critical cis-acting signals that define the element borders and act as transcriptional promoters [48], and we indeed found up to 5 -fold differences in the number of the ESTs corresponding to each type of element.

Among the "cut-and-paste" TEs in VdLs.17, the Tc1/ mariner superfamily, which derives its name from the founder transposons Tc1 of Caenorhabditis elegans and mariner of Drosophila mauritiana [8], was predominant. All 29 full-length DAHLIAE elements were approx. $2 \mathrm{~Kb}$ in size and comprised single intronless DDE_1-encoding transposases flanked by short (41-102 bp) TIRs. The DDE_1 transposases act generally as dimers or oligomers and harbor functional domains mediating protein-protein interaction, DNA-binding, -cleavage and -joining activities [49]. We predicted the presence of two types of N-terminal DNA-binding domains, HTH_psq and HTH_Tnp_Tc5, which are involved in the recognition and interaction with the TIRs [50]. The DDE_1 domain, first identified in bacterial transposases and retroviral integrases, is characterized by conservation of three aspartate (D) residues or two, non-contiguous (D) and one glutamate (E) residue, a catalytic triad that forms a pocket able to bind two divalent metal ions, mostly likely $\mathrm{Mg} 2+$, that are necessary for transposition [51]. The DDE signature has been detected in all eukaryotic "cut-and-paste" transposase superfamilies, indicating their common evolutionary origin [52]. In the DAHLIAE transposases, the three aspartate residues are separated by $110-112$ and 35 amino acids, respectively (D110-112D35D).
In vitro and in vivo trans-kingdom assays have previously demonstrated that, while host factors are not needed for transposition, intact transposase and TIRs are required for the initiation and completion of the process [53-56]. Tc1/mariner family transposon termini comprise at least three types of functional sequences involved in transposition: the 4-7 nucleotide TE cleavage sites at the outer extremities of the TIRs, the DRs within the TIRs, which are the transposase binding sites, and the UTRs, between the TIRs and ORFs, which act as enhancers of transposition efficiency [49,57-59]. Transposons of different families generally differ in their terminus structure and length, as well as in the transposase domain architecture. Each terminus/transposase combination appears to mediate a slightly different version of the "cut-and-paste" mobilization mechanism, ensuring transposition specificity [49]. In VdLs.17, while DAHLIAE3 starts with the unique cleavage motif CCCG and does not possess recognizable repeated sequences in the TIRs like those of Tc1/mariner TEs of other ascomycete fungi [60], DAHLIAE 1 and 2 start with the sequence ACGT-, and their TIRs contain two or three DRs. In particular, DAHLIAE2 carries three internal repetitions of a $17 \mathrm{bp}$-sequence at the $5^{\prime}$ terminus, and two at the 3' terminus. The DAHLIAE ORFs do not overlap with the TIR sequences and are flanked by asymmetric UTRs that vary in length from 33 to $125 \mathrm{bp}$, according to the TE family type. The VdLs.17 Activator and Mutator elements are highly degenerate (VdHATs) or with limited sequence similarities (VdMULEs). Most of the elements appeared to be of the non-autonomous type due to mutations that disrupted TIR sequences and/or resulted in incomplete transposases. Although these elements are probably unable to transpose and are fossils, we were able to identify corresponding ESTs for some of them. These sequences may therefore still play the important role of repressing transposition of the complete elements of the same family through transposase dilution or through a negative dominant repression by the truncated transposases.

Domestication is the process by which TE functional domains are incorporated into functional host proteins [5]. In VdLs.17 we found an insertion of a fragment of a Mutator element within a nitrilase gene. The fused sequence is predicted to generate a protein carrying inframe nitrilase and MULE domains. Although we did not find corresponding ESTs, we cannot rule out the possibility that this new protein is functional.

The EST data and expression analysis under heat stress further showed that several of the full length Class I and II TEs, which are predicted to code for complete transposition-mediating enzymes, are still transcriptionally active and differentially responsive to different stimuli. 
In general, our phylogenetic analysis of both Class I and II TEs mirrored the relationships among the fungal species that were defined by the fungal genome initiative on the basis of whole genome comparative studies. The evolutionary ancestors of the Copia, DAHLIAE, VdHAT and VdMULE TEs apparently evolved into different groups before insertion into the VdLs.17 genome. While high bootstrap values supported the monophyletic evolution of the $V$. dahliae Tc1/mariner and Mule elements, the Copia VdLTREs 1 and 5 and the VdHATs fell into distinct lineages. The three Gypsy families diversified after introgression into VdLs17 genome. Also, the Tc1/ mariner family DAHLIAE1 underwent a recent expansion in VdLs.17, generating five VdLs.17-specific subfamilies. These subfamilies differed in sequence and length of their termini, and of their ORF sequences. DAHLIAE1 a, b and d all putatively coded for intact transposases and were present in multiple, almost identical copies, comprising $74 \%$ of the total DNA TEs in VdLs.17. In fungi, the selective amplification of transposon subfamilies, such as we have detected for the DAHLIAE1 elements, has been associated to events of horizontal gene transfer [18], however no definitive mechanism has yet been proven.

It has been proposed that TEs may enhance recombination to cause genetic variation, giving populations the flexibility to adapt, a phenomenon which could be especially important for species that do not have a sexual phase [18]. TE clustering such as we observed in the VdLs.17 LS regions has been found in the genomes of other phytophathogenic fungi. In $M$. oryzae, for example, both Class I and II transposable elements are clustered within three regions of chromosome seven, characterized by a high rate of duplication and evolution [13]. Similarly, TEs were also found to cluster in regions undergoing rapid reorganization in the genome of the plant vascular pathogen $F$. oxysporum [61]. It has been hypothesized that these types of clusters are important for the generation and evolution of new genes [13], and it has been proposed that the transposon-rich LS regions in $V$. dahliae may impart a degree of genetic flexibility in the species, and allow rapid adaptation to new host niches [2]. The mechanism(s) by which TE clusters are generated in fungal genomes has not been elucidated yet. While the clustering could simply be the passive result of selection against TE incorporation into gene-rich zones of the genome, it alternatively could result from an active process related to TE function. Many TEs do selectively integrate into specific sequences [62,63], which could lead to biased TE distribution. Such selectivity has, for example, been observed for S. cerevisiae, in which Ty3 LTR elements are most often found to be integrated into upstream regions of genes transcribed by RNA polymerase III [64]. The parallel, non-random clustering of the Class I and II $V$. dahliae elements suggests that there may be synergistic interactions among different types of elements, and selective pressure in $V$. dahliae for TE clustering, which in turn may be important for generating the genomic diversity necessary for niche adaptation and host range expansion. Interestingly, among the 354 predicted genes encoded within the LS regions, there were no "housekeeping" genes [2]. Rather, the predicted genes encoded proteins of potential importance in pathogenicity, including bZIP transcription factors, ferric reductases, phospholipases, and other genes predicted to play a role in response to stress [2]. Moreover, clusters or pairs of genes were clearly duplicated in these LS regions ([2] and this study). It is unclear if such duplications are the direct result of TE activity, and more studies are required to investigate the role(s) of these putative pathogenicity factors. However, the presence of solo-LTR sequences does suggest that recombination between the repeated sequences of the LTR elements could be a contributing factor in the reorganization of Verticillium dahliae genome.

Since TEs can have a large effect on the genomes of their hosts, causing gene deletion and duplication as well as chromosomal rearrangements, host fitness could be adversely affected if TE-induced transposition and recombination events disrupted or altered function of essential genes. However, some filamentous fungi have a unique tool, known as repeat-induced point mutation (RIP), to deal with repetitive sequences such as TEs. RIP, a process first described in Neurospora crassa [22], was found to occur during the sexual cycle, and to subject duplicated sequences of $>400$ base pairs to irreversible CG to TA transition point mutations. Although RIP is known to only occur during the fungal sexual cycle, there are several examples in asexual fungi where TEs with RIP-like mutations have been identified $[23,65,66]$. The identification of RIP-like mutations in some VdLTRE2/3/4 sequences [2] indicated that, at some point in Verticillium spp. evolution, a RIP-like process operated to protect the genome from infiltration by TEs. The presence in $V$. dahliae and $V$. alboatrum of identifiable RID-like protein orthologs, which are known to be a part of the RIP machinery in $N$. crassa [67], suggests that these fungi may still possess the capacity for RIP. Interestingly, in VdLs.17, RIP mutations affected members of the Gypsy but not Copia superfamilies of retrotransposons, indicating either a differential susceptibility to RIP by different types of TEs or introgression by horizontal transfer of already RIPed sequences [2].

The TE content of different organisms is variable, sometimes accounting for as much as $60-80 \%$ of the genome, as in the case of cereals. More recently, mobile elements have been found to account for about half of 
the genome size also in the phytopathogenic oomycete Phytophthora infestans and the truffle fungus Tuber melanosporum [12]. Noticeably, a high degree of variability in the number and types of transposons has been documented even in closely related species. For instance, the TE content of the fungal plant pathogens $F$. oxysporum and $F$. graminearum differs by a two-fold order of magnitude, $4 \%$ and $0.03 \%$, respectively [68]. The observed TE spatial-temporal fluctuations appear to be TE-family dependent and governed by multiple mechanisms including elimination by ectopic recombination, extinction by genetic drift, reintroduction by horizontal transfer, and environmental stress-driven expansion (reviewed by Hua-Van et al. [12]). Despite the high level of identity (97\%) and synteny identified between the genomes of the recently sequenced $\mathrm{Vd}$ and $\mathrm{Va}$ isolates, the $V$. albo-atrum genome assembly was distinct in containing far less repetitive DNA than did that of $V$. dahliae [2]. In particular, VaMs.102 lacked the highly repetitive LS regions present in VdLs.17, and contained neither full-length nor defective VdLTRE5 Copia or "cut-and-paste" DNA elements. This absence seems simply to reflect the isolate of $V$. albo-atrum sequenced rather than a general feature of the species. In fact, $66 \%$ of the other $\mathrm{Va}$ isolates we surveyed for the presence of Vd.Ls17 TE-like sequences were positive for the Tc1/mariner DAHLIAE2.

Extensive studies conducted on natural populations of other organisms such as Drosophila and different plant species including Arabidopsis, barley, maize, rice and wheat have clearly demonstrated that transposon dynamics plays a central role also in generating intraspecific variability [69-73]. Our findings have shown that the Copia-like VdLTRE1 and the Gypsy-like retrotransposons are almost ubiquitous in $V$. dahliae, whereas the Copialike VdLTRE5 and most of the "cut-and-paste" DNA TEs appear to have a much more limited distribution. Although lack of detection of VdLs.17-like TEs could indeed reflect total absence of mobile elements, those Verticillium genomes without such elements may either contain related sequences that have diverged to a degree that prevented detection under the conditions used in this study (see Materials and Methods), or may simply harbor their own distinct arrays of transposable elements.

\section{Conclusions}

The identification and characterization of Class I and II TEs of Verticillium dahliae (VdLs.17) has allowed further exploration of the genetic diversity existing among the phytopathogenic Verticillium spp., and raised intriguing questions about the role(s) that TEs may have in shaping intra- and inter-specific evolution in this fungal genus. The discovery of chromosome location "hot spots" for TE insertion, as well as a "patchy" distribution among isolates of some of the TEs offers tantalizing evidence that TEs may play an important role in modulating genome architecture to allow Verticillium fungi to evolve quickly and adapt to their hosts, as has been suggested for other plant pathogens $[17,74]$. V. dahliae and $V$. albo-atrum were previously perceived, on the basis of vegetative compatibility and pathogenicity analyses, to have rather low genetic diversity. However, the results of molecular analyses carried out over the past two decades suggest that the species do in fact exhibit a high level of genetic variation and genome plasticity $[3,75,76]$. The analyses presented herein showed a broad range of different combinations of TE types in the different fungal strains, further supporting a major contribution of the transposable elements to the diversification of Verticillium spp. genomes.

\section{Methods}

\section{Bioinformatics and characterization of Verticillium Class I} and II transposons

The genome sequences of the fungal strains $V$. dahliae VdLs.17 and V. albo-atrum VaMs.102, and the associated expressed sequence tag (EST) information for strain VdLs.17 are available at www.broadinstitute.org/ annotation/genome/verticillium_dahliae/MultiHome.

$\mathrm{html}$ (Broad Institute), and served as the primary source for the TEs identified in this study. Virulence data and other information on these strains were previously published $[77,78]$. To identify repetitive sequences in the $V$. dahliae VdLS.17 and V. albo-atrum VaMs.102 genomes, sequences were searched using Cross_match [79], and filtering for alignments longer than $200 \mathrm{bp}$ with greater than $60 \%$ sequence similarity, as described in Klosterman et al. [2]. Class I and II transposon sequences were initially identified by searching among the repetitive sequences for putative transposon elements using computational predictions based on BLASTX analysis. For identification of the Class I elements, sequences from the above collection of putative transposon loci were manually inspected using the DNASTAR v. 6 software suite (DNASTAR Inc., Madison WI). Direct repeats flanking putative GAG and POL ORFs, a hallmark of Class I LTR elements, were first identified using the DNAstar program GeneQuest 6.1. Putative LTR and LINE-like element sequences were then aligned with ClustalW to identify type elements of the LTRcontaining Ty3/Gypsy, Ty1/Copia, and LINE-like class TEs. Conserved domain analysis of predicted open reading frames (ORFs) encoded by the putative LTR elements was conducted using the NCBI conserveddomain database search tool $[80,81]$, and the GenomeNet MOTIF search at http://motif.genome.jp. For the identification of the Class II "cut-and-paste" elements, 
sequences from the initial collection of putative transposon loci were manually inspected with homologybased methods including BLASTN searches of VdLs.17 and VaMs.102 genomes and NCBI databases. VdLs.17 genomic regions flanking the regions of homologies (3 to $6 \mathrm{~Kb}$ total) were then self-aligned using BLASTN align program to identify TIRs. Identification of DNA transposons was also based on use of the feature search tools available at the Verticillium group Database, in particular the feature type HMMR and the text search for DDE and hAT. We also searched the sequenced Verticillium genomes with complete or individual conserved domain aa sequences of well-characterized transposases of other closely related fungi. Nucleotide sequences of the identified full-length and defective VdLs.17 TEs were conceptually translated using TRANSLATE in ExPASy, and used in BLASTX searches to identify transposase conserved domains and the closest homologous sequences from other organisms.

\section{Phylogenetic analyses}

Nucleotide and amino acid sequences were aligned using the programs CLUSTALW and MUSCLE. Sequences were collapsed into haplotypes by manually removing INDELS using the multiple alignment editor program Jalview. Maximum-likelihood distance trees were inferred by using PhyML 3.0, and selecting the substitution models and at least 1000 bootstrap replications. Trees were graphically represented using the program TreeView. For the separation of the DAHLAIE1 elements into the subfamilies a to e, we used the TE fulllength nucleotide sequences (inclusive of TIRs). While the analysis of the Tc1/mariner transposases was based on complete protein sequences, the low level of conservation among VdHATs and VdMULEs allowed for an evaluation of relatedness based on the conserved hAT and MULE domains only. Conserved amino acid positions within each transposase functional domain were identified by visualizing their sequence alignments using the program MAFFT in Jalview.

\section{Fungal strains and growth conditions}

With the exception of V. albo-atrum isolates V104b and V4901 (provided by Dr. F. Daayf, University of Manitoba, Winnipeg MB), all Verticillium isolates used in this study (Table 3) are from culture collections of K.F.D, M. d.M.J. and P.V. Isolates were stored long-term at $-20^{\circ} \mathrm{C}$ as filter paper stock. Cultures were routinely grown at $24^{\circ} \mathrm{C}$ on complete medium (CM) agar and in CM broth [82].

\section{Nucleic acid isolation manipulations}

Fungal genomic DNA was isolated from Verticillium spores using a glass-bead breakage method as described previously [82]. For DNA blot hybridization, restriction enzyme-digested genomic DNA was electrophoretically size-fractionated through agarose gels, transferred by capillary blotting to positively charged nylon membranes (Roche Diagnostics, Indianapolis, IN), and fixed to the membrane by UV cross-linking. High stringency hybridizations $\left(65^{\circ} \mathrm{C}\right)$, and chemiluminescent detection of DIGlabeled hybrids were done as described previously [83]. Hybridization probes were synthesized from $V$. dahliae VdLs.17 gDNA by the incorporation of DIG-labeled dUTP into PCR amplification products. Amplification reactions contained Platinum Taq polymerase (Invitrogen, Canda Inc., Burlington, ON), DIG Labeling Mix (Roche Diagnostics, Indianapolis, IN), DNA, and forward and reverse primer pairs indicated in Additional file 9: Table S3. Reaction conditions involved an initial 2 min denaturation at $94^{\circ} \mathrm{C}$, followed by 30 amplification cycles: $94^{\circ} \mathrm{C}$ for $45 \mathrm{sec}, 65^{\circ} \mathrm{C}$ for $45 \mathrm{sec}, 72^{\circ} \mathrm{C}$ for $60 \mathrm{sec}$, and a final 5 min extension at $72^{\circ} \mathrm{C}$. PCR-based genomic survey for the presence of specific TEs in Vd and Va strains was performed using $20 \mathrm{ng}$ of genomic DNA in a $25 \mu \mathrm{L}$ reaction mixture containing $1.5 \mathrm{mM} \mathrm{MgCl}_{2}$, $100 \mathrm{mM}$ dNTPs, $100 \mathrm{pmol}$ of each TE-specific primer and 1 unit of Taq DNA polymersase (Promega). As controls of DNA equal loading and amplification specificity, we included in the experiments amplification of a VdLs.17 actin gene (VDAG_08445) and no-DNA samples. The sequences of the primers are listed in Additional file 9: Table S3.

\section{Transcriptional analysis}

The analysis of the expression of selected transposase genes was performed by semi-quantitative reverse transcription PCR (RT-PCR) experiments. Total RNA was extracted from VdLs.17 grown under control conditions $\left(25^{\circ} \mathrm{C}\right)$ or exposed to heat $\left(42^{\circ} \mathrm{C}\right)$ for 40 minutes using RNeasy Plant Mini Kit (QIAGEN). An in-column DNase step was added according to the manufacturer's instructions. First strand cDNA was synthetized using 500 ng total RNA, oligo (dT) and TaqMan Reverse Transcription kit (Applied Biosystems). PCR amplification were done using one $1 \mathrm{ul} \mathrm{cDNA}$ in $20 \mu \mathrm{l}$ reactions containing $1.5 \mathrm{mM} \mathrm{MgCl}, 100 \mathrm{mM}$ dNTPs, 100 pmol of each transposase-specific primer, 1 unit Taq DNA polymersase (Promega), and 30 amplification cycles. As controls of cDNA equal loading and amplification specificity, we included in the experiments amplification of cDNA corresponding to a VdLs.17 actin gene (VDAG_08445) and no-cDNA samples. The primers were designed on the basis of EST sequence information publicly available at the Verticillium Group Database/Fungal Genome Initiative/Broad Institute of MIT and Harvard (www.broadinstitute.org). Primer sequences are listed in the Additional file 9: Table S3. 


\section{Statistical analyses of transposon clustering in the genome of $V$. dahliae}

Odds ratio analyses [37] of transposons in the LS regions of the genome of isolate VdLs.17 of $V$. dahliae versus those encoded in the core genomic sequences of VdLs.17 were carried as described [2]. The only exception was that each Class I or II transposon type was parsed into numbers within LS regions or numbers in non-LS regions, and then grouped with the total numbers of genes within the LS regions or non LS regions for analyses. A total of 354 LS genes were used in the analyses and the total number of genes, determined by the Broad Institute annotation pipeline, was 10,535.

\section{Analyses of repeat-induced point mutation}

Sequences corresponding to each of the transposon families were identified by a BLASTN search of the $V$. dahliae genome $\left(\mathrm{E}\right.$ value cutoff $<1 \mathrm{E}^{-5}$ ) with the type elements. Sequences with a length of at least $500 \mathrm{bp}$ were then retrieved and aligned using MUSCLE [84], and analyzed using the RIPCAL software [44].

\section{Additonal files}

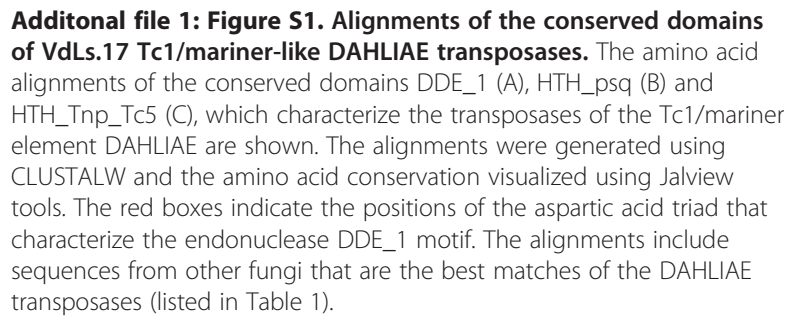

Additonal file 2: Figure S2. Terminal inverted repeats (TIRs) of the Class II elements identified in the VdLs.17 genome. The TIR

nucleotide sequences of the Tc1/mariner-like elements DAHLAIE 1 and 2 (A), the Activator elements VdHAT 1 and 2 (B) and of the Mutator elements VdMULE 1 to 3 are shown. The internal direct duplications are underlined. The asterisks indicate mismatches.

Additonal file 3: Figure S3. Alignment of VdLs.17 hAT and MULE transposase domains. The amino acid alignments used for the phylogenetic analysis of the VdHAT (A) and VdMULE (B) elements are shown. The alignments were generated using CLUSTALW, all gaps were removed manually and the amino acid conservation visualized using Jalview tools.

Additional file 4: Table S1. Activator-and Mutator- like transposons identified in the VdLs.17 genome.

Additional file 5: Table S2. Odd ratio analysis of Class I and Class II transposon domain encoded in the genome of VdLs.17.

Additonal file 6: Figure S4. Analysis of the expression of VdLs. 17 Class II transposases in response to heat stress. The transcription of the indicated Class II transposases was analyzed by RT-PCR experiments using cDNA synthetized from total RNA extracted from VdLs.17 grown at $25^{\circ} \mathrm{C}$ or exposed to heat stress $\left(42^{\circ} \mathrm{C}\right.$ for 45 minutes). The primers used to amplify the transposase ORFs were designed on the basis of the sequence of the corresponding ESTs publicly available at the Verticillium Group Database (www.broadinstitute.org/annotation/genome/ verticillium_dahliae/MultiHome.html) (Primer sequences are listed in Supplemental Table 3).

Additonal file 7: Figure S5. RIPCAL analysis of VdLs.17 Class II TEs.
Search of the indicated DNA transposon sequences for evidence of RIP mutations by RIPCAL analyses did not show any clear bias in the type of mutations observed. Analysis of VdLTRE2-like sequences, in which a bias for CA to TA transition mutation was previously identified [2] was included in our study as a positive control of RIPing.

Additonal file 8: Figure S6. Distribution of Class I and II elements identified in the Vd.Ls17 genome in other phytopathogenic Verticillium spp. isolates. A) Southern hybridization analysis of the indicated $\mathrm{Vd}$ and $\mathrm{Va}$ fungal isolates for the presence of retrotransposons. Genomic DNA was digested with EcoRI (top panel), Bg/ll (middle panel), or Sacl (bottom panel), and blots hybridized with DIG-labelled probes corresponding to VdLTRE1, VdLTRE2/3/4, or VdLTRE5, respectively. Sizes of DIG-labelled molecular weight markers $(\mathrm{Kb})$ are indicated to the right of the images; B) PCR amplifications of retrotransposons in the indicated Vd and Va isolates, using VdLTRE1, VdLTRE2 and VdLTRE5 primer pairs. Amplification of actin was used as control (not shown); FSDW, notemplate reaction; C) PCR amplification of DAHLIAE, VdHAT and VdMULE elements was obtained by using as a template genomic DNA (20 ng) of the indicated $\mathrm{Vd}$ and $\mathrm{Va}$ isolates; D) To verify specificity of the bands of different size obtained from amplification of DAHLIAE 1d, PCR products were blotted onto nylon membranes and hybridized with DIG-labelled specific probes. Details of the isolates used in the survey are provided in Table 3. Sequences of the primers used in the survey are listed in Supplemental Table 3 .

Additional file 9: Table S3. List of PCR amplification primers used in this study.

\section{Competing interests}

The authors declare no competing financial interests.

\section{Author's contributions}

S.G.A. and X.T. identified and characterized retrotransposons and DNA transposons, respectively; K.P. and S.G.A. contributed to the survey of Vd and VA isolates for the presence of TEs, and K.P. to the RT-PCR expression studies; M.d.M.J. contributed to the survey of $\mathrm{Vd}$ and $\mathrm{VA}$ for the presence of DNA TES; S.J.K. and L.J.M. contributed to the analysis of the TE distribution within the VdLs.17 sequenced genome; L.J.M. identified the candidate transposons based on bioinformatic analysis; K.F.D. and P.V. conceived the study, and contributed to the transposon discovery and characterization. All authors contributed to the writing and critical revision of the manuscript, and read and approved the final manuscript.

\section{Acknowledgements}

We are grateful to Dr. R. Dean for his valuable critique of the manuscript. Thanks go also to Dr. F. Daayf for providing V. albo-atrum isolates, Mr. A. Molnar for graphics assistance, and Ms. S. Grant for technical assistance. The research was supported through the Broad Fungal Genome Initiative, with funds to PV from the NSF/USDA Microbial Genome Sequencing Program's U. S. Department of Agriculture's Cooperative State Research Education and Extension Service, and to KFD from a Natural Sciences and Engineering Research Council of Canada Discovery Grant, and Agriculture and Agri-Food Canada.

\section{Author details}

${ }^{1}$ University of Western Ontario, London, ON, Canada. ${ }^{2}$ Department of Plant Pathology, North Carolina State University, Raleigh, NC, USA. ${ }^{3}$ Department of Plant Pathology, Penn State University, University Park, PA, USA. ${ }^{4}$ USDA-ARS, Salinas, CA, USA. ${ }^{5}$ University of Massachusetts, Amherst, MA, USA.

${ }^{6}$ Agriculture and Agri-Food Canada, London, ON, Canada.

Received: 22 February 2012 Accepted: 30 May 2012

Published: 16 July 2012

\section{References}

1. Pegg GF, Brady BL: Verticillium wilts, Verticillium wilts. New York: CABI Publishing; 2002

2. Klosterman SJ, Subbarao KV, Kang S, Veronese P, Gold SE, Thomma BPHJ, Chen Z, Henrissat B, Lee Y-H, Park J, Garcia-Pedrajas MD, Barbara DJ, Anchieta A, de Jonge R, Santhanam P, Maruthachalam K, Atallah Z, 
Amyotte SG, Paz Z, Inderbitzin P, Hayes RJ, Heiman DI, Young S, Zeng Q Engels R, Galagan J, Cuomo CA, Dobinson KF, Ma L-J: Comparative genomics yields insights into niche adaptation of plant vascular wilt pathogens. PLoS Pathogens 2011, 7:e1002137.

3. Dobinson KF, Patterson NA, White GJ, Grant S: DNA fingerprinting and vegetative compatibility analysis indicate multiple origins for Verticillium dahliae race 2 tomato isolates from Ontario, Canada. Mycol Res 1998, 102:1089-1095.

4. Usami T, Shishido M, Ebihara Y, Kamigahira Y, Amemiya Y: Retrotransposonlike elements in the genome of Verticillium dahliae may be used as DNA markers for fungal species and pathotypes. J Gen Plant Pathol 2005, 71:117-123.

5. Feshotte $\mathrm{C}$ : The contribution of transposable elements to the evolution of regulatory networks. Nat Rev Genet 2008, 9:397-405.

6. Bourque $\mathrm{G}$ : Transposable elements in gene regulation and in the evolution of vertebrate genomes. Curr Opinion Gen \& Dev 2009, 19:607-612.

7. Shapiro JA: Mobile DNA and evolution in the 21th century. Mob DNA 2010, 1:1-14.

8. Wicker T, Sabot F, Hua-Van A, Bennetzen JL, Capy P, Chalhoub B, Flavell A, Leroy P, Morgante M, Panaud O, Paux E, SanMiguel P, Schulman AH: A unified classification system for eukaryotic transposable elements. Nat Rev Genet 2007, 8:973-982.

9. Kapitonov W, Jurka J: A universal classification of eukaryotic transposable elements implemented in Repbase. Nat Rev Genet 2008, 9:411-412.

10. Kapitonov W, Jurka J: Rolling-circle transposons in eukaryotes. Proc Natl Acad Sci USA 2001, 98:8714-8719.

11. Pritham EJ, Putliwala T, Feschotte Cd: Mavericks, a novel class of giant transposable elements widespread in eukaryotes and related to DNA viruses. Gene 2007, 390:3-17.

12. Hua-Van A, Le Rouzic A, Boutin TS, Filee J, Capy P: The struggle for life of the genome's selfish architects. Biol Direct 2011, 6:19-48.

13. Thon M, Pan H, Diener S, Papalas J, Taro A, Mitchell T, Dean R: The role of transposable element clusters in genome evolution and loss of synteny in the rice blast fungus Magnaporthe oryzae. Genome Biol 2006, 7:R16.

14. Braumann I, van den Berg MA, Kempken F: Strain-specific retrotransposonmediated recombination in commercially used Aspergillus niger strain. Mol Genet Genom 2008, 280:319-325.

15. Panaccione DG, Pitkin JW, Walton JD, Annis SL: Transposon-like sequences at the TOX2 locus of the plant-pathogenic fungus Cochliobolus carbonum. Gene 1996, 176:103-109.

16. Hatta R, Ito K, Hosaki Y, Tanaka T, Tanaka A, Yamamoto M, Akimitsu K, Tsuge T: A conditionally dispensable chromosome controls host-specific pathogenicity in the fungal plant pathogen Alternaria alternata. Genetic 2002, 161:59-70.

17. Dean RA, Talbot NJ, Ebbole DJ, Farman ML, Mitchell TK, Orbach MJ, Thon M, Kulkarni R, Xu J-R, Pan H, Read ND, Lee Y-H, Carbone I, Brown D, Oh YY, Donofrio N, Jeong JS, Soanes DM, Djonovic S, Kolomiets E, Rehmeyer C, Li W, Harding M, Kim S, Lebrun M-H, Bohnert H, Coughlan S, Butler J, Calvo S, Ma L-J, Nicol R, Purcell S, Nusbaum C, Galagan JE, Birren BW: The genome sequence of the rice blast fungus Magnaporthe grisea. Nature 2005, 434:980-986.

18. Daboussi MJ, Capy P: Transposable elements in filamentous fungi. Annu Rev Microbiol 2003, 57:275-299.

19. Aravin AA, Hannon GJ, Brennecke J: The Piwi-piRNA pathway provides an adaptive defense in the transposon arms race. Science 2007, 318:761-764.

20. Lisch D: Epigenetic regulation of transposable elements in plants. Annu Rev Plant Biol 2009, 60:43-66.

21. Galagan JE, Selker EU: RIP: the evolutionary cost of genome defense. Trends Genet 2004, 20:417-423.

22. Cambareri E, Jensen B, Schabtach E, Selker E: Repeat-induced G-C to A-T mutations in Neurospora. Science 1989, 244:1571-1575.

23. Braumann I, Van den Berg M, Kempken F: Repeat induced point mutation in two asexual fungi, Aspergillus niger and Penicillium chrysogenum. Curr Genet 2008, 53:287-297.

24. Harrell L, Melcher U, Atkins JF: Predominance of six different hexanucleotide recoding signals 3 ' of read-through stop codons. NuC Acids Res 2002, 30:2011-2017.

25. Konieczny A, Voytas DF, Cummings MP, Ausubel FM: A superfamily of Arabidopsis thaliana retrotransposons. Genetics 1991, 127:801-809.
26. Kim JM, Vanguri S, Boeke JD, Gabriel A, Voytas DF: Transposable elements and genome organization: a comprehensive survey of retrotransposons revealed by the complete Saccharomyces cerevisiae genome sequence. Genome Res 1998, 8:464-478.

27. Marin I, Llorens C: Ty3/Gypsy retrotransposons:description of new Arabidopsis thaliana elements and evolutionary perspectives derived from comparative genomic data. Mol Biol Evol 2000, 17:1040-1049.

28. Zhu P, Oudemans PV: A long terminal repeat retrotransposon Cgret from the phytopathogenic fungus Colletotrichum gloeosporioides on cranberry. Curr Genet 2000, 38:241-247.

29. Bouvet GF, Jacobi V, Bernier L: Characterization of three DNA transposons in the Dutch elm disease fungi and evidence of repeat-induced point (RIP) mutations. Fungal Genet Biol 2007, 44:430-443.

30. Kempken $F$, Windhofer $F$ : The hAT family: a versatile transposon group common to plants, fungi, animals, and man. Chromosoma 2001, 110:1-9.

31. Linder-Basso D, Foglia R, Zhu P, Hillman Bl: Crypt1, an active Ac-like transposon from the chestnut blight fungus, Cryphonectria parasitica. Mol Genet Genom 2001, 265:730-738.

32. Rep M, van der Does HC, Cornelissen BJC: Drifter, a novel, low copy hAT-like transposon in Fusarium oxysporum is activated during starvation. Fungal Genet Biol 2005, 42:546-553.

33. Okuda M, Ikeda K, Namiki F, Nishi K, Tsuge T: Tfo1: an Ac-like transposon from the plant pathogenic fungus Fusarium oxysporum. Mol Gen Genet 1998, 258:599-607.

34. Kempken F, Kuck U: Restless, an active Ac-like transposon from the fungus Tolypocladium inflatum: Structure, expression, and alternative RNA splicing. Mol Cell Biol 1996, 16:6563-6572.

35. Gao Q, Jin K, Ying S-H, Zhang Y, Xiao G, Shang Y, Duan Z, Hu X, Xie X-Q, Zhou G, Peng G, Luo Z, Huang W, Wang B, Fang W, Wang S, Zhong Y, Ma L-J, St Leger RJ, Zhao G-P, Pei Y, Feng M-G, Xia Y, Wang C: Genome sequencing and comparative transcriptomics of the model entomopathogenic fungi Metarhizium anisopliae and M. acridum. PLoS Genet 2011, 7:e1001264.

36. Chalvet F, Grimaldi C, Kaper F, Langin T, Daboussi MJ: Hop, an active Mutator-like element in the genome of the fungus Fusarium oxysporum. Mol Biol Evol 2003, 20:1362-1375.

37. Sokal RR, Rohlf FJ: Biometry, the principles and practice of statistics in biological research, analysis of frequencies. 3rd edition. New York: W.H. Freeman and Company; 1995.

38. Ikeda K, Nakayashiki H, Takagi M, Tosa Y, Mayama S: Heat shock, copper sulfate and oxidative stress activate the retrotransposon MAGGY resident in the plant pathogenic fungus Magnaporthe grisea. Mol Genet Genom 2001, 266:318-325.

39. Ogasawara H, Obata H, Hata Y, Takahashi S, Gomi K: Crawler, a novel Tc1/mariner-type transposable element in Aspergillus oryzae transposes under stress conditions. Fungal Genet Biol 2009, 46:441-449.

40. Anava N, Roncero MIG: Skippy, a retrotransposon from the fungal plant pathogen Fusarium oxysporum. Mol Gen Genet 1995, 249:637-647.

41. Levis C, Fortini D, Brygoo Y: Flipper, a mobile Fot1-like transposable element in Botrytis cinerea. Mol Gen Genet 1997, 254:674-680.

42. Daboussi MJ, Langin T, Brygoo Y: Fot1, a new family of fungal transposable elements. Mol Gen Genet 1992, 232:12-16.

43. Enkerli J, Bhatt G, Covert SF: Nht1, a transposable element cloned from a dispensable chromosome in Nectria haematococca. Mol Plant Microb Interac 1997, 10:742-749.

44. Hane J, Oliver R: RIPCAL: a tool for alignment-based analysis of repeat-induced point mutations in fungal genomic sequences. $B M C$ Bioinforma 2008, 9:478.

45. Joaquim TR, Rowe RC: Reassessment of vegetative compatibility relationships among strains of Verticillium dahliae using nitrate-nonutilizing mutants. Phytopathology 1990, 80:1160-1166.

46. Gao X, Havecker ER, Baranov PV, Atkins JF, Voytas DF: Translational recoding signals between gag and pol in diverse LTR retrotransposons. RNA 2003, 9:1422-1430.

47. Forbes E, Nieduszynska S, Brunton F, Gibson J, Glover LA, Stansfield Control of gag-pol gene expression in the Candida albicans retrotransposon Tca2. BMC Mol Biol 2007, 8:94.

48. Lauermann V, Hermankova M, Boeke JD: Increased length of long terminal repeats inhibits Ty1 transposition and leads to the formation of tandem multimers. Genetics 1997, 145:911-922. 
49. Brillet B, Benjamin B, Bigot $Y$, Yves B, Auge-Gouillou C, Corinne A-G: Assembly of the Tc1 and mariner transposition initiation complexes depends on the origins of their transposase DNA binding domains. Genetica 2007, 130:105-120.

50. Tudor M, Lobocka M, Goodell M, Pettitt J, Ohare K: The pogo transposable element family of Drosophila melanogaster. Mol Gen Genet 1992 232:126-134.

51. Hickman $A B$, Chandler M, Dyda F: Integrating prokaryotes and eukaryotes: DNA transposases in light of structure. Crit Rev Biochem Mol Biol 2010, 45:50-69.

52. Yuan YW, Wessler SR: The catalytic domain of all eukaryotic cut-and-paste transposase superfamilies. Proc Natl Acad Sci USA 2011, 108:7884-7889.

53. Vos JC, DeBaere I, Plasterk RHA: Transposase is the only nematode protein required for in vitro transposition of Tc1. Genes Dev 1996, 10:755-761.

54. Gueiros-Filho FJ, Beverley SM: Development of a heterologous transposon system for Leishmania. Memorias Instituto Oswaldo Cruz 1997, 92:16

55. Fadool JM, Hartl DL, Dowling JE: Transposition of the mariner element from Drosophila mauritiana in zebrafish. Proc Natl Acad Sci USA 1998, 95:5182-5186

56. Sherman A, Dawson A, Mather C, Gilhooley H, Li Y, Mitchell R, Finnegan D, Sang $\mathrm{H}$ : Transposition of the Drosophila element mariner into the chicken germ line. Nat Biotechnol 1998, 16:1050-1053.

57. Tosi LRO, Beverley SM: Cis and trans factors affecting Mos1 mariner evolution and transposition in vitro, and its potential for functional genomics. Nucl Acids Res 2000, 28:784-790.

58. Pledger DW, Fu YQ, Coates CJ: Analyses of cis-acting elements that affect the transposition of Mos1 mariner transposons in vivo. Mol Genet Genom 2004, 272:67-75.

59. Casteret S, Chbab N, Cambefort J, Auge-Gouillou C, Bigot Y, Rouleux-Bonnin F: Physical properties of DNA components affecting the transposition efficiency of the mariner Mos1 element. Mol Genet Genom 2009, 282:531-546

60. Marini M, Zanforlin T, Santos P, Barros R, Guerra A, Puccia R, Felipe M, Brigido M, Soares C, Ruiz J, Silveira J, Cisalpino P: Identification and characterization of Tc1/mariner-like DNA transposons in genomes of the pathogenic fungi of the Paracoccidioides species complex. BMC Genomics 2010, 11:130.

61. Hua-Van A, Daviere J-M, Kaper F, Langin T, Daboussi M-J: Genome organization in Fusarium oxysporum: clusters of class II transposons. Curr Genet 2000, 37:339-347.

62. Seringhaus M, Kumar A, Hartigan J, Snyder M, Gerstein M: Genomic analysis of insertion behavior and target specificity of mini-Tn7 and Tn3 transposons in Saccharomyces cerevisiae. Nucl Acids Res 2006, 34:e57.

63. Kondrychyn I, Garcia-Lecea M, Emelyanov A, Parinov S, Korzh V: Genomewide analysis of Tol2 transposon reintegration in zebrafish. BMC Genomics 2009, 10:418.

64. Voytas DF, Boeke JD: Yeast retrotransposons and tRNAs. Trends Genet 1993, 9:421-427.

65. Crouch JA, Glasheen BM, Giunta MA, Clarke BB, Hillman BI: The evolution of transposon repeat-induced point mutation in the genome of Colletotrichum cereale: Reconciling sex, recombination and homoplasy in an "asexual" pathogen. Fungal Genet Biol 2008, 45:190-206.

66. Montiel MD, Lee HA, Archer DB: Evidence of RIP (repeat-induced point mutation) in transposase sequences of Aspergillus oryzae. Fungal Genet Biol 2006, 43:439-445.

67. Freitag M, Williams RL, Kothe GO, Selker EU: A cytosine methyltransferase homologue is essential for repeat-induced point mutation in Neurospora crassa. Proc Natl Acad Sci USA 2002, 99:8802-8807.

68. Ma LJ, van der Does HC, Borkovich KA, Coleman JJ, Daboussi MJ, Di Pietro A, Dufresne M, Freitag M, Grabherr M, Henrissat B, Houterman PM, Kang S, Shim WB, Woloshuk C, Xie XH, Xu JR, Antoniw J, Baker SE, Bluhm BH, Breakspear A, Brown DW, Butchko RAE, Chapman S, Coulson R, Coutinho PM, Danchin EGJ, Diener A, Gale LR, Gardiner DM, Goff S, Hammond-Kosack KE, Hilburn K, Hua-Van A, Jonkers W, Kazan K, Kodira CD, Koehrsen M, Kumar L, Lee YH, Li LD, Manners JM, Miranda-Saavedra D, Mukherjee M, Park G, Park J, Park SY, Proctor RH, Regev A, Ruiz-Roldan MC, Sain D, Sakthikumar S, Sykes S, Schwartz DC, Turgeon BG, Wapinski I, Yoder O, Young S, Zeng QD, Zhou SG, Galagan J, Cuomo CA, Kistler HC, Rep M: Comparative genomics reveals mobile pathogenicity chromosomes in Fusarium. Nature 2010, 464:367-373.
69. Belyayev A, Kalendar R, Brodsky L, Nevo E, Schulman A, Raskina O: Transposable elements in a marginal plant population: temporal fluctuations provide new insights into genome evolution of wild diploid wheat. Mob DNA 2010, 1:6.

70. Bennetzen $J L$, Ramakrishna W: Exceptional haplotype variation in maize. Proc Natl Acad Sci USA 2002, 99:9093-9095.

71. Huang X, Lu G, Zhao Q, Liu X, Han B: Genome-wide analysis of transposon insertion polymorphisms reveals intraspecific variation in cultivated rice. Plant Physiol 2008, 148:25-40.

72. Picot S, Wallau GL, Loreto ELS, Heredia FO, Hua-Van A, Capy P: The mariner transposable element in natural populations of Drosophila simulans. Heredity 2008, 101:53-59

73. Wright SI, Le QH, Schoen DJ, Bureau TE: Population dynamics of an Ac-like transposable element in self- and cross-pollinating Arabidopsis. Genetics 2001, 158:1279-1288.

74. Favarol LCdL, Araujoll WLd, Azevedolll JLd, Paccola-Meirelles LD: The biology and potential for genetic research of transposable elements in filamentous fungi. Genet Mol Biol 2005, 28:804-813.

75. Klosterman SJ, Atallah ZK, Vallad GE, Subbarao KV: Diversity, pathogenicity, and management of Verticillium species. Annu Rev Phytopathol 2009, 47:39-62.

76. Atallah ZK, Maruthachalam K, du Toit L, Koike ST, Davis RM, Klosterman SJ, Hayes RJ, Subbarao KV: Population analyses of the vascular plant pathogen Verticillium dahliae detect recombination and transcontinental gene flow. Fungal Genet Biol 2010, 47:416-422

77. Bhat RG, Subbarao KV: Host range specificity in Verticillium dahliae. Phytopathology 1999, 89:1218-1225.

78. Qin QM, Vallad GE, Wu BM, Subbarao KV: Phylogenetic analyses of phytopathogenic isolates of Verticillium spp. Phytopathology 2006, 96:582-592.

79. Ewing B, Hillier L, Wendl MC, Green P: Base-calling of automated sequencer traces using Phred. I. Accuracy assessment. Genome Res 1998 8:175-185.

80. Marchler-Bauer A, Bryant SH: CD-Search: protein domain annotations on the fly. Nucl Acids Res 2004, 32:W327-W331.

81. Marchler-Bauer A, Anderson JB, Chitsaz F, Derbyshire MK, DeWeese-Scott C, Fong JH, Geer LY, Geer RC, Gonzales NR, Gwadz M, He S, Hurwitz DI, Jackson JD, Ke Z, Lanczycki CJ, Liebert CA, Liu C, Lu F, Lu S, Marchler GH, Mullokandov M, Song JS, Tasneem A, Thanki N, Yamashita RA, Zhang D, Zhang N, Bryant SH: CDD: specific functional annotation with the Conserved Domain Database. Nucl Acids Res 2009, 37:D205-D210.

82. Dobinson KF: Genetic transformation of the vascular wilt fungus Verticillium dahliae. Can J Bot 1995, 73:710-715.

83. Klimes A, Dobinson KF: A hydrophobin gene, $\mathrm{VDH} 1$, is involved in microsclerotial development and spore viability in the plant pathogen Verticillium dahliae. Fungal Genet Biol 2006, 43:283-294.

84. Edgar R: MUSCLE: a multiple sequence alignment method with reduced time and space complexity. BMC Bioinforma 2004, 5:113.

\section{doi:10.1186/1471-2164-13-314}

Cite this article as: Amyotte et al:: Transposable elements in phytopathogenic Verticillium spp.: insights into genome evolution and inter- and intra-specific diversification. BMC Genomics 2012 13:314.

\section{Submit your next manuscript to BioMed Central and take full advantage of:}

- Convenient online submission

- Thorough peer review

- No space constraints or color figure charges

- Immediate publication on acceptance

- Inclusion in PubMed, CAS, Scopus and Google Scholar

- Research which is freely available for redistribution 